\title{
FIRST PASSAGE TIMES FOR MARKOV ADDITIVE PROCESSES WITH POSITIVE JUMPS OF PHASE TYPE
}

\author{
LOTHAR BREUER, ${ }^{*}$ University of Kent
}

\begin{abstract}
The present paper generalises some results for spectrally negative Lévy processes to the setting of Markov additive processes (MAPs). A prominent role is assumed by the first passage times, which will be determined in terms of their Laplace transforms. These have the form of a phase-type distribution, with a rate matrix that can be regarded as an inverse function of the cumulant matrix. A numerically stable iteration to compute this matrix is given. The theory is first developed for MAPs without positive jumps and then extended to include positive jumps having phase-type distributions. Numerical and analytical examples show agreement with existing results in special cases.
\end{abstract}

Keywords: Lévy process; first passage time; supremum; Markov additive process; reflected process; stationary distribution

2000 Mathematics Subject Classification: Primary 60J25

Secondary 60G51; 60J55

\section{Introduction}

Markov modulation is the basis for many generalisations in stochastic processes. It leads from renewal processes to Markov renewal theory, which has enabled an analysis of the classical M/G/1 and GI/M/1 queues. Another important example for queueing theory is the Markov-modulated Poisson process, later generalised to Markovian arrival processes, which are now standard use in current literature. On a more general level, Markov additive processes, introduced in [10], [13], and [14], are a powerful generalisation of Lévy processes. The use of Markov additive processes (MAPs) or, rather, subclasses of MAPs, has become increasingly popular in stochastic modelling. For a recent textbook presentation, see [3, Chapter XI].

Stochastic modelling based on MAPs requires a theory which is able to provide methods for the computation of key characteristics such as first passage times, suprema, or stationary distributions. On the level of Lévy processes, many results can be obtained for the spectrally negative variant, i.e. the class of Lévy processes without positive jumps. Standard references are [8, Chapter VII] or [9]. Recently, some results have been proven for Lévy processes with phase-type positive jumps as well; see [5], [11], [19], or [20]. In the present paper we firstly generalise some of these results to the level of MAPs without positive jumps and then we sketch an extension to MAPs with phase-type positive jumps. Related works investigating MAPs include [12, Chapter 14], [17], and [18].

Many arguments in this paper are straightforward generalisations of ideas from [1], in which the overall supremum of MAPs with continuous paths was investigated. As expected, the main method in generalising the existing theory of Lévy processes consists in finding a matrix

Received 21 August 2007; revision received 30 April 2008.

* Postal address: Institute of Mathematics and Statistics, University of Kent, Canterbury CT2 7NF, UK.

Email address: 1.breuer@kent.ac.uk 
equivalent to the inverse function of the cumulant. It turns out that the Laplace transform of a first passage time has a phase-type form where we need to determine two matrices: the rate matrix $\boldsymbol{U}(\gamma)$ and the recovery matrix $\boldsymbol{A}(\gamma)$. To this aim, we derive fixed point equations for $\boldsymbol{A}(\gamma)$ and $\boldsymbol{U}(\gamma)$ from which these can be determined via successive approximation.

An extension of the classical fluctuation theory for spectrally negative Lévy processes is the incorporation of positive jumps having phase-type distributions. The phase-type assumption is almost without loss of generality due to the following two results. Asmussen et al. [5, Proposition 1] showed that Lévy processes with phase-type jumps are dense within the class of one-dimensional Lévy processes (equipped with the Prokhorov distance). Furthermore, Pistorius [20, Proposition 1] showed that the joint distribution of first passage times and the respective overshoots (equipped with convergence in distribution) are continuous functionals of Lévy processes (equipped with convergence in law). In Section 5 we shall sketch an analogous extension for MAPs.

As far as the author is aware, the only existing approach to determine the Laplace transform of first passage times for MAPs is the construction of suitable martingales, as proposed in [4] or [16]. One advantage of the present paper is seen in the feature that here the classical theory of Lévy processes finds a natural extension. A switch of analytical methods is not required.

In Section 2 some notation and preliminary results shall be provided. Initially, results for first passage times will be derived in Section 3. The question of numerically computing the matrices $\boldsymbol{A}(\gamma)$ and $\boldsymbol{U}(\gamma)$ will be examined in Section 4. An extension of the theory, incorporating positive jumps of phase type, is described in Section 5. The relation between first passage times, suprema, and the stationary distribution for the process reflected at the origin carries through to the MAP level and shall be described briefly in Section 6. In Section 7 we investigate the relation between the martingale approach of Asmussen and Kella [4] and a diagonalisation of $\boldsymbol{U}(\gamma)$ in the case where no negative subordinators are part of the MAP. The last section contains some numerical examples, which show that in some special cases the results obtained here are in agreement with the results obtained by different approaches.

\section{Preliminaries}

\subsection{Lévy processes}

A Lévy process is a real-valued stochastic process with stationary and independent increments. The study of this class of processes goes back as far as the foundations of modern probability theory. A modern textbook is [8], whereas [9] gives an extensive presentation for the fluctuation theory of spectrally negative Lévy processes. For ease of reference, we shall restate some classical results for these, along with the necessary notation, in the following.

Let $y=\left(Y_{t}: t \geq 0\right)$ be a one-dimensional real-valued Lévy process with parameters $\mu$ for the drift, $\sigma^{2}$ for the variation, and $v$ for its Lévy measure. Assume that $y$ has no positive jumps, i.e. that $v$ is concentrated on $(-\infty, 0]$. Then the Lévy-Khintchine formula yields $\mathrm{E}\left(\exp \left(\alpha Y_{t}\right)\right)=\exp (t \psi(\alpha))$ for $\operatorname{Re}(\alpha) \geq 0$, where the function

$$
\psi(\alpha)=\alpha \mu+\frac{1}{2} \sigma^{2} \alpha^{2}+\int_{-\infty}^{0}\left(\mathrm{e}^{\alpha x}-1-\alpha x 1_{\{x>-1\}}\right) v(\mathrm{~d} x)
$$

(where $1_{\{.\}}$is the indicator function) is called the Laplace exponent of $\mathcal{y}_{\text {; }} \mathrm{cf}$. [8, Section VII.1]. A subordinator is a Lévy process with increasing paths, implying that $\sigma^{2}=0$ and that the Lévy measure is concentrated on $[0, \infty)$. We assume that $y$ is not the negative of a subordinator. Furthermore, let $Y_{0}=0$ throughout this section. 
Since the function $\psi$ is strictly convex, there is a largest solution, called $\Phi(0)$, of the equation $\psi(\alpha)=0, \alpha \geq 0$. As $y$ is not the negative of a subordinator, it follows that $\lim _{\alpha \rightarrow \infty} \psi(\alpha)=\infty$. Hence, there is an inverse function of $\psi:[\Phi(0), \infty) \rightarrow[0, \infty)$. It shall be denoted by $\Phi:[0, \infty) \rightarrow[\Phi(0), \infty)$. Denote the running maximum of $\mathcal{y}$ until time $t \geq 0$ by $S_{t}:=\sup \left\{Y_{s}: s \leq t\right\}$. The assumption that $y$ has no positive jumps yields the following lemmas.

Lemma 1. ([8, Theorem VII.1].) Let $\tau(x):=\inf \left\{s \geq 0: Y_{s}>x\right\}$ for $x \geq 0$ denote the first passage time of the level $x$. Then $(\tau(x): x \geq 0)$ is a subordinator with Laplace exponent $\Phi$.

Lemma 2. ([8, Corollary VII.2].) Let $\mathscr{E}(\lambda) \sim \operatorname{Exp}(\lambda)$ be an exponentially distributed random variable, independent of $\mathcal{y}$ and with $\lambda>0$. Then $S_{\mathcal{E}(\lambda)}$ has an exponential distribution with parameter $\Phi(\lambda)$.

Lemma 3. ([8, Theorem VII.4].) Let $\mathcal{E}(q) \sim \operatorname{Exp}(q)$ be an exponentially distributed random variable, independent of $\mathcal{y}_{\text {and with }} q>0$. Furthermore, let $G_{\mathcal{E}(q)}=\sup \left\{t<\mathcal{E}(q): X_{t}=S_{t}\right\}$. Then

$$
\begin{gathered}
\mathrm{E}\left(\exp \left(-\alpha G_{\mathcal{E}(q)}-\beta S_{\mathcal{E}(q)}\right)\right)=\frac{\Phi(q)}{\Phi(\alpha+q)+\beta}, \\
\mathrm{E}\left(\exp \left(-\alpha\left(\mathcal{E}(q)-G_{\mathcal{E}(q)}\right)-\beta\left(S_{\mathcal{E}(q)}-Y_{\mathcal{E}(q)}\right)\right)\right)=\frac{q(\Phi(\alpha+q)-\beta)}{\Phi(q)(\alpha+q-\psi(\beta))},
\end{gathered}
$$

for $\alpha, \beta>0$.

Remark 1. Later on, we shall use the second equation of Lemma 3 with a matrix argument $\boldsymbol{U}$ instead of $-\beta$. Of course, convergence of $\mathrm{E}\left(\exp \left(-\alpha\left(\mathcal{E}(q)-G_{\mathcal{E}(q)}\right) \boldsymbol{I}+\boldsymbol{U}\left(S_{\mathcal{E}(q)}-Y_{\mathcal{E}_{(q)}}\right)\right)\right)$ can be expected only for matrices $\boldsymbol{U}$ with all eigenvalues having negative real part.

\subsection{MAPs}

Let $\mathcal{g}=\left(J_{t}: t \geq 0\right)$ be an irreducible Markov (jump) process with finite state space $E=\{1, \ldots, m\}$ and infinitesimal generator matrix $\boldsymbol{Q}=\left(q_{i j}\right)_{i, j \in E}$. We call $J_{t}$ the phase at time $t \geq 0$. Define the real-valued process $X=\left(X_{t}: t \geq 0\right)$ as evolving like a Lévy process with parameters $\mu_{i}$ (drift), $\sigma_{i}^{2}$ (variation), and $v_{i}$ (Lévy measure) during intervals when the phase equals $i \in E$. Whenever $\mathcal{g}$ jumps from state $i \in E$ to another state $j \in E$, this may be accompanied (with probability $\lambda_{i j}^{-}$) by a jump of $\mathcal{X}$ which has distribution function $F_{i j}$. Then the two-dimensional process $(\mathcal{X}, \mathcal{g})$ is called a MAP.

A MAP can also be defined by the following property (see [3, Section XI.2a]): $(\mathcal{X}, \mathcal{g})$ is a Markov process such that

$$
\mathrm{E}\left(f\left(X_{t+s}-X_{t}\right) g\left(J_{t+s}\right) \mid \mathcal{F}_{t}, J_{t}=i\right)=\mathrm{E}\left(f\left(X_{s}\right) g\left(J_{s}\right) \mid X_{0}=0, J_{0}=i\right)
$$

holds for all $s, t>0$ and $i \in E$, where $f$ and $g$ are measurable functions.

First we shall investigate only MAPs without positive jumps, for which the measures $v_{i}$ and $F_{i j}$ have support on the negative real numbers only. Then the cumulant of the Lévy process $\chi^{(i)}$ in phase $i$ may be written as

$$
\psi_{i}(\alpha)=\alpha \mu_{i}+\frac{1}{2} \sigma_{i}^{2} \alpha^{2}+\int_{-\infty}^{0}\left(\mathrm{e}^{\alpha x}-1-\alpha x 1_{\{x>-1\}}\right) v_{i}(\mathrm{~d} x)
$$

for such $\alpha \in \mathbb{C}$ that allow an integration. For notational convenience, we define the modified distribution functions $\tilde{F}_{i j}$ by

$$
\tilde{F}_{i j}(0)=1-\lambda_{i j}^{-} \quad \text { and } \quad \tilde{F}_{i j}(\mathrm{~d} x)=\lambda_{i j}^{-} F_{i j}(-\mathrm{d} x)
$$


for $x>0$ and $i, j \in E$. Note that $\lambda_{i i}=0$, which implies that $\tilde{F}_{i i}=\delta_{0}$, denoting the Dirac one-point measure on 0 .

Denote the matrix-valued moment generating function of $X_{t}$, given $X_{0}=0$, by $\hat{\boldsymbol{X}}_{t}(\alpha)$. Write $\hat{\boldsymbol{F}}(s)=\left(\hat{F}_{i j}(s)\right)_{i, j \in E}$, where $\hat{F}_{i j}(s)=\int_{0}^{\infty} \mathrm{e}^{s x} \mathrm{~d} \tilde{F}_{i j}(x)$ is the moment generating function of $\tilde{F}_{i j}$. By Proposition XI.2.2 of [3], the $(i, j)$ th entry of $\hat{\boldsymbol{X}}_{t}(\alpha)$ is given as

$$
\mathrm{E}\left(\exp \left(\alpha X_{t}\right) ; J_{t}=j \mid X_{0}=0, J_{0}=i\right)=\left(\mathrm{e}^{K(\alpha) t}\right)_{i j}
$$

with

$$
\boldsymbol{K}(\alpha)=\operatorname{diag}\left(\psi_{i}(\alpha)\right)_{i \in E}+\boldsymbol{Q} \circ \hat{\boldsymbol{F}}(-\alpha)
$$

for arguments $\alpha \in \mathbb{C}$ that allow convergence of the integrals involved. Here the notation $\boldsymbol{A} \circ \boldsymbol{B}$ denotes entrywise multiplication of the matrices $\boldsymbol{A}$ and $\boldsymbol{B}$ having the same dimension, i.e. $(\boldsymbol{A} \circ \boldsymbol{B})_{i j}:=a_{i j} b_{i j}$.

\section{First passage times}

Let $\left(\left(X_{t}, J_{t}\right): t \geq 0\right)$ be a MAP with phase space $E=E_{-} \cup E_{\sigma}$, where the subspaces have the following meaning: during any phase $i \in E_{-}$, the level process $X^{(i)}$ is the negative of a subordinator or the constant process $X_{t}^{(i)}=0$ for all $t \geq 0$. The subspace $E_{\sigma}$ contains all other phases, i.e. for $i \in E_{\sigma}$, the level process is not a negative subordinator.

The analysis of positive jumps will be included later in Section 5, so that we may first assume that all Lévy processes $\mathcal{X}^{(i)}$ with $i \in E_{\sigma}$ are spectrally negative. As negative subordinators, the $\chi^{(i)}$ for $i \in E_{-}$are spectrally negative anyway. Then the level process is upper semicontinuous.

Denote the time of first passage beyond the level $x \geq 0$ by $\tau(x):=\inf \left\{t \geq 0: X_{t}>x\right\}$. Whenever we start from a negative level $X_{0}<0$, we write $\tau:=\tau(0)$ for the first passage time beyond 0 . Define the functions

$$
f_{i j}(x):=\mathrm{E}_{i j}\left(\mathrm{e}^{-\gamma \tau} \mid X_{0}=x\right):=\mathrm{E}\left(\mathrm{e}^{-\gamma \tau} ; J_{\tau}=j \mid J_{0}=i, X_{0}=x\right)
$$

for all $x<0$ and an arbitrary but fixed $\gamma \geq 0$. Since the paths of $\mathcal{X}$ are upper semicontinuous and spatially homogeneous (conditioned on the phase process), we obtain the functional equation

$$
f_{i j}(x+y)=\sum_{k \in E_{\sigma}} f_{i k}(x) f_{k j}(y) \quad \text { for all } x, y \leq 0 .
$$

Spatial homogeneity of $\mathcal{X}$ (conditioned on the phase process) yields, for $x>0$ and $i, j \in E$,

$$
\begin{aligned}
\mathrm{E}_{i j}\left(\mathrm{e}^{-\gamma \tau(x)}\right) & :=\mathrm{E}\left(\mathrm{e}^{-\gamma \tau(x)} ; J_{\tau(x)}=j \mid J_{0}=i, X_{0}=0\right) \\
& =\mathrm{E}_{i j}\left(\mathrm{e}^{-\gamma \tau(0)} \mid X_{0}=-x\right) \\
& =f_{i j}(-x) .
\end{aligned}
$$

Let $\mathrm{E}_{(-, \sigma)}\left(\mathrm{e}^{-\gamma \tau(x)}\right)$ denote the matrix with entries $\mathrm{E}_{i j}\left(\mathrm{e}^{-\gamma \tau(x)}\right)$ over the range $i \in E_{-}$and $j \in E_{\sigma}$. Likewise, $\mathrm{E}_{(\sigma, \sigma)}\left(\mathrm{e}^{-\gamma \tau(x)}\right)$ shall contain these entries over the range $i, j \in E_{\sigma}$. Then (3) yields the form

$$
\mathrm{E}_{(-, \sigma)}\left(\mathrm{e}^{-\gamma \tau(x)}\right)=\boldsymbol{A}(\gamma) \mathrm{e}^{\boldsymbol{U}(\gamma) x} \quad \text { and } \quad \mathrm{E}_{(\sigma, \sigma)}\left(\mathrm{e}^{-\gamma \tau(x)}\right)=\mathrm{e}^{\boldsymbol{U}(\gamma) x}
$$

for some subgenerator matrix $\boldsymbol{U}(\gamma)$ and a subtransition matrix $\boldsymbol{A}(\gamma)$. The different forms in (4) are due to the boundary condition

$$
\mathrm{E}_{i j}\left(\mathrm{e}^{-\gamma \tau(0)}\right)= \begin{cases}\delta_{i j}, & i, j \in E_{\sigma}, \\ a_{i j}(\gamma), & i \in E_{-}, j \in E_{\sigma},\end{cases}
$$


where $\delta_{i j}$ denotes the Kronecker function (i.e. $\delta_{i j}=1$ if $i=j$ and $\delta_{i j}=0$ otherwise) and

$$
a_{i j}(\gamma):=\mathrm{E}\left(\mathrm{e}^{-\gamma \tau(0)}, J_{\tau(0)}=j \mid X_{0}=0, J_{0}=i\right)
$$

The values $a_{i j}(\gamma)$ are nonnegative but strictly less than 1 if $\gamma>0$ and $\mathcal{X}^{(i)}$ is a negative subordinator. Let $\boldsymbol{A}(\gamma)$ denote the $E_{-} \times E_{\sigma}$ matrix with entries $a_{i j}(\gamma)$. It is clear that $\boldsymbol{A}(\gamma)$ is a subtransition matrix, i.e. it has nonnegative entries and its row sums are at most 1.

Since a first passage to a level above cannot occur in a negative subordinator phase, we obtain $\mathrm{P}\left(J_{\tau(x)}=j\right)=0$ for all $j \in E_{-}$and, thus, $\mathrm{E}_{(-,-)}\left(\mathrm{e}^{-\gamma \tau(x)}\right)=\mathrm{E}_{(\sigma,-)}\left(\mathrm{e}^{-\gamma \tau(x)}\right)=\mathbf{0}$, where $\mathbf{0}$ denotes a zero matrix of suitable dimension. Altogether this yields

$$
\mathrm{E}\left(\mathrm{e}^{-\gamma \tau(x)}\right)=\left(\begin{array}{cc}
\mathrm{e}^{\boldsymbol{U}(\gamma) x} & \mathbf{0} \\
\boldsymbol{A}(\gamma) \mathrm{e}^{\boldsymbol{U}(\gamma) x} & \mathbf{0}
\end{array}\right)=\left(\begin{array}{c}
\boldsymbol{I}_{\sigma} \\
\boldsymbol{A}(\gamma)
\end{array}\right)\left(\begin{array}{ll}
\mathrm{e}^{\boldsymbol{U}(\gamma) x} & \mathbf{0}
\end{array}\right),
$$

where $\boldsymbol{I}_{\sigma}$ denotes the identity matrix of dimension $\left|E_{\sigma}\right|$.

It remains to determine $\boldsymbol{U}(\gamma)$ and $\boldsymbol{A}(\gamma)$, which is of course the main part of the paper. Since clearly $f_{i j}(x) \rightarrow 0$ as $x \rightarrow-\infty$ (for all $i, j \in E$ ), we see from (4) that all eigenvalues of $\boldsymbol{U}(\gamma)$ must have negative real part.

Denote the semigroup of transition operators for $(\mathcal{X}, \mathcal{g})$ by $\left(P_{t}: t \geq 0\right)$, and define the matrix-valued function $\boldsymbol{f}(x)=\left(f_{i j}(x)\right)_{i, j \in E}$. By definition, $P_{t} \boldsymbol{f}(x)=\mathrm{E}\left(\mathrm{e}^{-\gamma(\tau-t)} \mid X_{0}=x\right)$ for $x<0$ and small enough $t$. This yields, for the infinitesimal generator $\mathcal{A}$ of $(\mathcal{X}, \mathcal{g})$,

$$
\begin{aligned}
\mathcal{A} \boldsymbol{f} & =\lim _{t \downarrow 0} \frac{1}{t}\left(P_{t} \boldsymbol{f}-\boldsymbol{f}\right) \\
& =\lim _{t \downarrow 0} \frac{1}{t}\left(\mathrm{E}\left(\mathrm{e}^{-\gamma(\tau-t)}\right)-\mathrm{E}\left(\mathrm{e}^{-\gamma \tau}\right)\right) \\
& =\mathrm{E}\left(\mathrm{e}^{-\gamma \tau}\right) \lim _{t \downarrow 0} \frac{1}{t}\left(\mathrm{e}^{\gamma t \boldsymbol{I}}-\boldsymbol{I}\right) \\
& =\mathrm{E}\left(\mathrm{e}^{-\gamma \tau}\right) \gamma \boldsymbol{I} \\
& =\gamma \boldsymbol{f},
\end{aligned}
$$

where $\mathrm{E}\left(\mathrm{e}^{-\gamma \tau}\right)=\left(\mathrm{E}_{i j}\left(\mathrm{e}^{-\gamma \tau} \mid X_{0}=\cdot\right)\right)_{i, j \in E}$. On the other hand, we obtain, for $i \in E$ and $j \in E_{\sigma}$, where $\boldsymbol{e}_{i}^{\prime}$ and $\boldsymbol{e}_{j}$ denote row and column base vectors of dimension $|E|$ and $\left|E_{\sigma}\right|$, respectively,

$$
\begin{aligned}
\mathcal{A} f_{i j}(x)= & \mathcal{A}\left(\boldsymbol{e}_{i}^{\prime}\left(\begin{array}{c}
\boldsymbol{I}_{\sigma} \\
\boldsymbol{A}(\gamma)
\end{array}\right) \mathrm{e}^{-\boldsymbol{U}(\gamma) x} \boldsymbol{e}_{j}\right) \\
= & \boldsymbol{e}_{i}^{\prime}\left(\begin{array}{c}
\boldsymbol{I}_{\sigma} \\
\boldsymbol{A}(\gamma)
\end{array}\right)\left(\frac{1}{2} \sigma_{i}^{2} \boldsymbol{U}(\gamma)^{2}-\mu_{i} \boldsymbol{U}(\gamma)\right) \mathrm{e}^{-\boldsymbol{U}(\gamma) x} \boldsymbol{e}_{j} \\
& +\boldsymbol{e}_{i}^{\prime}\left(\begin{array}{c}
\boldsymbol{I}_{\sigma} \\
\boldsymbol{A}(\gamma)
\end{array}\right) \int_{-\infty}^{0}\left(\mathrm{e}^{-\boldsymbol{U}(\gamma) z}-\boldsymbol{I}+\boldsymbol{U}(\gamma) z 1_{\{z>-1\}}\right) \nu_{i}(\mathrm{~d} z) \mathrm{e}^{-\boldsymbol{U}(\gamma) x} \boldsymbol{e}_{j} \\
& +\sum_{k \in E} q_{i k} \boldsymbol{e}_{k}^{\prime}\left(\begin{array}{c}
\boldsymbol{I}_{\sigma} \\
\boldsymbol{A}(\gamma)
\end{array}\right) \int_{0}^{\infty} \mathrm{e}^{\boldsymbol{U}(\gamma) z} \mathrm{~d} \tilde{F}_{i k}(z) \mathrm{e}^{-\boldsymbol{U}(\gamma) x} \boldsymbol{e}_{j},
\end{aligned}
$$

with the definitions $\tilde{F}_{i j}(0)=1-\lambda_{i j}^{-}$and $\tilde{F}_{i j}(\mathrm{~d} y)=\lambda_{i j}^{-} F_{i j}(-\mathrm{d} y)$ for $i, j \in E$. Note that 
$\lambda_{i i}^{-}=0$ such that $\tilde{F}_{i i}=\delta_{0}$ is the Dirac (one-point) measure on 0 . This leads to

$$
\begin{aligned}
\gamma \boldsymbol{e}_{i}^{\prime} & \left(\begin{array}{c}
\boldsymbol{I}_{\sigma} \\
\boldsymbol{A}(\gamma)
\end{array}\right) \mathrm{e}^{-\boldsymbol{U}(\gamma) x} \boldsymbol{e}_{j} \\
& =\gamma f_{i j}(x) \\
& =\mathcal{A} f_{i j}(x) \\
& =\boldsymbol{e}_{i}^{\prime}\left(\left(\begin{array}{c}
\boldsymbol{I}_{\sigma} \\
\boldsymbol{A}(\gamma)
\end{array}\right) \psi_{i}(-\boldsymbol{U}(\gamma))+\int_{0}^{\infty} \boldsymbol{Q} \circ \tilde{\boldsymbol{F}}(\mathrm{d} z)\left(\begin{array}{c}
\boldsymbol{I}_{\sigma} \\
\boldsymbol{A}(\gamma)
\end{array}\right) \mathrm{e}^{\boldsymbol{U}(\gamma) z}\right) \mathrm{e}^{-\boldsymbol{U}(\gamma) x} \boldsymbol{e}_{j}
\end{aligned}
$$

for all $x<0$ and $j \in E_{\sigma}$, where the $(i, j)$ th entry of the matrix $\boldsymbol{Q} \circ \tilde{\boldsymbol{F}}$ is the measure $q_{i j} \tilde{F}_{i j}$. Thus, a first result for $\boldsymbol{U}(\gamma)$ and $\boldsymbol{A}(\gamma)$ is

$$
\gamma \boldsymbol{e}_{i}^{\prime}\left(\begin{array}{c}
\boldsymbol{I}_{\sigma} \\
\boldsymbol{A}(\gamma)
\end{array}\right)=\boldsymbol{e}_{i}^{\prime}\left(\left(\begin{array}{c}
\boldsymbol{I}_{\sigma} \\
\boldsymbol{A}(\gamma)
\end{array}\right) \psi_{i}(-\boldsymbol{U}(\gamma))+\int_{0}^{\infty} \boldsymbol{Q} \circ \tilde{\boldsymbol{F}}(\mathrm{d} z)\left(\begin{array}{c}
\boldsymbol{I}_{\sigma} \\
\boldsymbol{A}(\gamma)
\end{array}\right) \mathrm{e}^{\boldsymbol{U}(\gamma) z}\right)
$$

for all $i \in E$. We can write this in matrix form as

$$
\begin{aligned}
\gamma\left(\begin{array}{c}
\boldsymbol{I}_{\sigma} \\
\boldsymbol{A}(\gamma)
\end{array}\right)= & \boldsymbol{\Delta}_{\sigma^{2} / 2}\left(\begin{array}{c}
\boldsymbol{I}_{\sigma} \\
\boldsymbol{A}(\gamma)
\end{array}\right) \boldsymbol{U}(\gamma)^{2}+\boldsymbol{\Delta}_{\mu}\left(\begin{array}{c}
\boldsymbol{I}_{\sigma} \\
\boldsymbol{A}(\gamma)
\end{array}\right) \boldsymbol{U}(\gamma) \\
& +\int_{-\infty}^{0} \boldsymbol{\Delta}_{v}(\mathrm{~d} z)\left(\begin{array}{c}
\boldsymbol{I}_{\sigma} \\
\boldsymbol{A}(\gamma)
\end{array}\right)\left(\mathrm{e}^{-\boldsymbol{U}(\gamma) z}-\boldsymbol{I}+\boldsymbol{U}(\gamma) z 1_{\{z>-1\}}\right) \\
& +\int_{0}^{\infty} \boldsymbol{Q} \circ \tilde{\boldsymbol{F}}(\mathrm{d} z)\left(\begin{array}{c}
\boldsymbol{I}_{\sigma} \\
\boldsymbol{A}(\gamma)
\end{array}\right) \mathrm{e}^{\boldsymbol{U}(\gamma) z}
\end{aligned}
$$

with $\boldsymbol{\Delta}_{\mu}:=\operatorname{diag}\left(\mu_{1}, \ldots, \mu_{m}\right)$, where $m=|E|$ is the number of phases and, analogously, for $\boldsymbol{\Delta}_{\sigma^{2} / 2}$ and $\boldsymbol{\Delta}_{\nu}$, the entries of the latter being the Lévy measures.

Remark 2. For the case without negative subordinators, i.e. $E=E_{\sigma}$ or $E_{-}=\varnothing$, there is no matrix $\boldsymbol{A}(\gamma)$ and we find the following interpretation for the matrix $-\boldsymbol{U}(\gamma)$. Looking at (1), we see that the function $\boldsymbol{K}$ takes the role of the cumulant function $\psi$ for a Lévy process. The matrix $-\boldsymbol{U}(\gamma)$ generalises the inverse function $\psi^{-1}$, which is denoted by $\phi$ in the present paper. This interpretation is supported by the following observation. For $i \in E_{\sigma}$, the $i$ th row in (2) is

$$
\boldsymbol{e}_{i}^{\prime} \boldsymbol{K}(\alpha)=\boldsymbol{e}_{i}^{\prime} \psi_{i}(\alpha)+\left(q_{i j} \hat{F}_{i j}(-\alpha)\right)_{j \in E}=\boldsymbol{e}_{i}^{\prime} \psi_{i}(\alpha)+\sum_{j \in E} q_{i j} \boldsymbol{e}_{j}^{\prime} \hat{F}_{i j}(-\alpha),
$$

where $\hat{F}_{i j}(s)=\int_{0}^{\infty} \mathrm{e}^{s x} \mathrm{~d} \tilde{F}_{i j}(x)$. Substituting $-\boldsymbol{U}(\gamma)$ for $\alpha$ yields

$$
\boldsymbol{e}_{i}^{\prime} \boldsymbol{K}(-\boldsymbol{U}(\gamma))=\boldsymbol{e}_{i}^{\prime} \psi_{i}(-\boldsymbol{U}(\gamma))+\sum_{j \in E} q_{i j} \boldsymbol{e}_{j}^{\prime} \hat{F}_{i j}(\boldsymbol{U}(\gamma))=\gamma \boldsymbol{e}_{i}^{\prime} \quad \text { for all } i \in E,
$$

where the last equality is due to (5). In this sense, $-\boldsymbol{U}$ is the inverse function of the cumulant matrix $\boldsymbol{K}$.

Example 1. For $\gamma=0$ and $\sigma_{i}>0$ for all $i \in E$ (i.e. $E_{-}=\varnothing$ and there is no matrix $\boldsymbol{A}(\gamma)$ ), (5) has been derived before. The case without jumps (i.e. $\tilde{F}_{i j}=\delta_{0}$ for all $i, j \in E$ ) has been examined in [1]. Here we obtain

$$
\psi_{i}(-\boldsymbol{U}(0))=-\mu_{i} \boldsymbol{U}(0)+\frac{\sigma_{i}^{2}}{2} \boldsymbol{U}(0)^{2} \quad \text { and } \quad \int_{0}^{\infty} \boldsymbol{Q} \circ \tilde{\boldsymbol{F}}(\mathrm{d} z) \mathrm{e}^{\boldsymbol{U}(0) z}=\boldsymbol{Q} .
$$


This reduces (5) to Equation (4.1) of [1], where the notation is $r_{i}=\mu_{i}, \Lambda=\boldsymbol{Q}$, and $U=\boldsymbol{U}(0)$. Adding possible downward jumps upon phase changes leads to Equation (5.4) of [18], which is given in a more adapted way as Equation (6) of [17]. Another special case appears in [20, Proposition 2(i)]. Here we have $\gamma=0, \mu_{i}=\sigma(i) c+v(i), \sigma_{i}^{2}=\sigma(i)^{2} s^{2}$, and $v_{i}=\sigma(i) v$. Furthermore, there are no jumps upon epochs of phase changes.

\section{Computation of the matrices $U(\gamma)$ and $A(\gamma)$}

Although (6) looks like a nice equation to determine $\boldsymbol{A}(\gamma)$ and $\boldsymbol{U}(\gamma)$, there is no obvious numerical approach to solve it. The next theorems provide an iteration which seems numerically stable.

For all phases $i \in E_{\sigma}$, let $\phi_{i}(0)$ be the largest solution of the equation $\psi_{i}(\alpha)=0, \alpha \geq 0$; cf. [8, Section VII] or Section 2.1. Denote the inverse functions of $\psi_{i}:\left[\phi_{i}(0), \infty\right) \rightarrow[0, \infty)$ by $\phi_{i}$. Define, for a square matrix $\boldsymbol{U}$, the functions

$$
\hat{F}_{i k}(\boldsymbol{U})=\int_{0}^{\infty} \mathrm{e}^{\boldsymbol{U} y} \mathrm{~d} \tilde{F}_{i k}(y),
$$

where $\tilde{F}_{i k}(0)=1-\lambda_{i k}^{-}$and $\tilde{F}_{i k}(\mathrm{~d} y)=\lambda_{i k}^{-} F_{i k}(-\mathrm{d} y)$ for all $i, k \in E$, and

$$
L_{i}(-\boldsymbol{U})=\frac{q_{i}}{\phi_{i}\left(q_{i}\right)} \cdot\left(\phi_{i}\left(q_{i}+\gamma\right) \boldsymbol{I}+\boldsymbol{U}\right) \cdot\left(\left(q_{i}+\gamma\right) \boldsymbol{I}-\psi_{i}(-\boldsymbol{U})\right)^{-1} \quad \text { for all } i \in E_{\sigma} .
$$

Theorem 1. The matrices $\boldsymbol{A}(\gamma)$ and $\boldsymbol{U}(\gamma)$ satisfy the following fixed point equations:

$$
\boldsymbol{e}_{i}^{\prime} \boldsymbol{U}(\gamma)=-\phi_{i}\left(q_{i}+\gamma\right) \boldsymbol{e}_{i}^{\prime}+\phi_{i}\left(q_{i}\right) \sum_{k \in E} p_{i k} \boldsymbol{e}_{k}^{\prime}\left(\begin{array}{c}
\boldsymbol{I}_{\sigma} \\
\boldsymbol{A}(\gamma)
\end{array}\right) \hat{F}_{i k}(\boldsymbol{U}(\gamma)) L_{i}(-\boldsymbol{U}(\gamma))
$$

for all $i \in E_{\sigma}$ and

$$
\boldsymbol{e}_{i}^{\prime} \boldsymbol{A}(\gamma)=\sum_{k \neq i} q_{i k} \boldsymbol{e}_{k}^{\prime}\left(\begin{array}{c}
\boldsymbol{I}_{\sigma} \\
\boldsymbol{A}(\gamma)
\end{array}\right) \hat{F}_{i k}(\boldsymbol{U}(\gamma))\left(\left(q_{i}+\gamma\right) \boldsymbol{I}_{\sigma}-\psi_{i}(-\boldsymbol{U}(\gamma))\right)^{-1} \quad \text { for } i \in E_{-} .
$$

Proof. The proof is a straightforward generalisation of the proofs of Theorems 3.1 and 4.1 of [1]. First we explain (9). Let $J_{0}=i$ for some $i \in E_{\sigma}$. Let $M^{i}$ denote the maximum and let $G^{i}$ denote the time to the maximum before the first phase change. The first equation in Lemma 3 shows that

$$
\mathrm{E}\left(\exp \left(-\gamma G^{i}\right) ; M^{i} \in \mathrm{d} x\right)=\phi_{i}\left(q_{i}\right) \exp \left(-\phi_{i}\left(q_{i}+\gamma\right) \cdot x\right) \mathrm{d} x .
$$

Furthermore, we consider the measure

$$
\mathrm{d} F_{Y^{i}}(y):=\mathrm{E}\left(\exp \left(-\gamma\left(\tau_{i}-G^{i}\right)\right) ; M^{i}-X_{\tau_{i}-} \in \mathrm{d} y\right),
$$

where $\tau_{i}$ is the time until the first phase change. Once the maximum $M^{i}$ is reached, the discounted height process $\mathrm{e}^{-\gamma t} X_{t}$ descends by $y$ units according to the distribution function $F_{Y^{i}}$, whereafter $\mathcal{g}$ jumps to another phase $k$ with probability $p_{i k}$. This may be accompanied by another downward jump ( $z$ units) having distribution function $\tilde{F}_{i k}$. From there, the ascend goes beyond $M^{i}$ again with time-discounted probability

$$
\boldsymbol{e}_{k}^{\prime}\left(\begin{array}{c}
\boldsymbol{I}_{\sigma} \\
\boldsymbol{A}(\gamma)
\end{array}\right) \mathrm{e}^{\boldsymbol{U}(\gamma)(y+z)}
$$


Altogether this leads to the equations

$$
\begin{aligned}
U_{i j}(\gamma)= & -\phi_{i}\left(q_{i}+\gamma\right) \delta_{i j} \\
& +\phi_{i}\left(q_{i}\right) \int_{0}^{\infty} \mathrm{d} F_{Y^{i}}(y) \sum_{k \in E} p_{i k} \int_{0}^{\infty} \mathrm{d} \tilde{F}_{i k}(z) \boldsymbol{e}_{k}^{\prime}\left(\begin{array}{c}
\boldsymbol{I}_{\sigma} \\
\boldsymbol{A}(\gamma)
\end{array}\right) \mathrm{e}^{\boldsymbol{U}(\gamma)(y+z)} \boldsymbol{e}_{j} \\
= & -\phi_{i}\left(q_{i}+\gamma\right) \delta_{i j} \\
& +\phi_{i}\left(q_{i}\right) \sum_{k \in E} p_{i k} \boldsymbol{e}_{k}^{\prime}\left(\begin{array}{c}
\boldsymbol{I}_{\sigma} \\
\boldsymbol{A}(\gamma)
\end{array}\right) \int_{0}^{\infty} \mathrm{e}^{\boldsymbol{U}(\gamma) z} \mathrm{~d} \tilde{F}_{i k}(z) \int_{0}^{\infty} \mathrm{e}^{\boldsymbol{U}(\gamma) y} \mathrm{~d} F_{Y^{i}}(y) \boldsymbol{e}_{j}
\end{aligned}
$$

for $i, j \in E_{\sigma}$. Since $\boldsymbol{U}(\gamma)$ has only eigenvalues with negative real part, the second equation in Lemma 3 can be applied to the argument $-\boldsymbol{U}(\gamma)$ instead of the scalar $\beta>0$. This yields

$$
U_{i j}(\gamma)=-\phi_{i}\left(q_{i}+\gamma\right) \delta_{i j}+\phi_{i}\left(q_{i}\right) \sum_{k \in E} p_{i k} \boldsymbol{e}_{k}^{\prime}\left(\begin{array}{c}
\boldsymbol{I}_{\sigma} \\
\boldsymbol{A}(\gamma)
\end{array}\right) \hat{F}_{i k}(\boldsymbol{U}(\gamma)) L_{i}(-\boldsymbol{U}(\gamma)) \boldsymbol{e}_{j}
$$

for all $i, j \in E_{\sigma}$. The statement is just the row vector version of the above equalities.

Now the explanation of (10). Denote the distribution function of $-X_{t-}$ by $\tilde{F}_{X_{t}}$. Starting in a descending phase $i \in E_{-}$, we obtain, for $i \in E_{-}$and $j \in E_{\sigma}$,

$$
\begin{array}{r}
A_{i j}(\gamma)=\mathrm{E}\left(\mathrm{e}^{-\gamma \tau(0)}, J_{\tau(0)}=j \mid X_{0}=0, J_{0}=i\right) \\
=\int_{0}^{\infty} q_{i} \exp \left(-q_{i} t\right) \mathrm{e}^{-\gamma t} \sum_{k \neq i} p_{i k} \boldsymbol{e}_{k}^{\prime}\left(\begin{array}{c}
\boldsymbol{I}_{\sigma} \\
\boldsymbol{A}(\gamma)
\end{array}\right) \\
\quad \times \int_{0}^{\infty} \int_{0}^{\infty} \mathrm{e}^{\boldsymbol{U}(\gamma)(z+x)} \mathrm{d} \tilde{F}_{i k}(z) \mathrm{d} \tilde{F}_{X_{t}}(x) \boldsymbol{e}_{j} \mathrm{~d} t,
\end{array}
$$

which follows from (4) by conditioning on the time $t$ of the first phase change. Since, before time $t$, we are exclusively in phase $i$, we may apply the Lévy-Khintchine formula for $\mathcal{X}^{(i)}$, which yields

$$
\int_{0}^{\infty} \mathrm{e}^{\boldsymbol{U}(\gamma) x} \mathrm{~d} \tilde{F}_{X_{t}}(x)=\mathrm{E}\left(\exp \left(\boldsymbol{U}(\gamma)\left(-X_{t}\right)\right)\right)=\mathrm{E}\left(\exp \left(-\boldsymbol{U}(\gamma) X_{t}\right)\right)=\exp \left(\psi_{i}(-\boldsymbol{U}(\gamma)) t\right)
$$

for any $t>0$. Hence, in (11) we obtain

$$
A_{i j}(\gamma)=\sum_{k \neq i} q_{i k} \boldsymbol{e}_{k}^{\prime}\left(\begin{array}{c}
\boldsymbol{I}_{\sigma} \\
\boldsymbol{A}(\gamma)
\end{array}\right) \hat{F}_{i k}(\boldsymbol{U}(\gamma)) \int_{0}^{\infty} \exp \left(-\left(q_{i}+\gamma\right) t\right) \exp \left(\psi_{i}(-\boldsymbol{U}(\gamma)) t\right) \mathrm{d} t \boldsymbol{e}_{j} .
$$

It remains to show that the integral occuring herein converges. From (12) we see that the matrix $\exp \left(\psi_{i}(-\boldsymbol{U}(\gamma)) t\right)$ is substochastic since $\left(-X_{t}\right)$ is nonnegative and $\boldsymbol{U}(\gamma)$ is a subgenerator. Thus, for all $t \geq 0$, its entries are nonnegative and bounded by 1 . Since $q_{i}+\gamma>0$, this ascertains convergence of the integral and, hence, the statement.

Remark 3. To illustrate the relation between Theorem 1 and (5), we show that the two statements are equivalent, given that the matrices $\phi_{i}\left(q_{i}+\gamma\right) \boldsymbol{I}+\boldsymbol{U}(\gamma)$ and $\left(q_{i}+\gamma\right) \boldsymbol{I}-\psi_{i}(-\boldsymbol{U}(\gamma))$ are invertible for all $i \in E_{\sigma}$. This yields an alternative proof of Theorem 1. It has been shown at the end of the above proof that the matrices $\left(q_{i}+\gamma\right) \boldsymbol{I}-\psi_{i}(-\boldsymbol{U}(\gamma))$ are invertible for $i \in E_{-}$. 
Starting from Theorem 1, we obtain, for $i \in E_{\sigma}$,

$$
\begin{aligned}
& \boldsymbol{e}_{i}^{\prime} \boldsymbol{U}(\gamma)\left(\left(q_{i}+\gamma\right) \boldsymbol{I}-\psi_{i}(-\boldsymbol{U}(\gamma))\right) \\
& \quad=-\phi_{i}\left(q_{i}+\gamma\right) \boldsymbol{e}_{i}^{\prime}\left(\left(q_{i}+\gamma\right) \boldsymbol{I}-\psi_{i}(-\boldsymbol{U}(\gamma))\right) \\
& \quad+q_{i} \sum_{k \in E} p_{i k} \boldsymbol{e}_{k}^{\prime}\left(\begin{array}{c}
\boldsymbol{I}_{\sigma} \\
\boldsymbol{A}(\gamma)
\end{array}\right) \hat{F}_{i k}(\boldsymbol{U}(\gamma))\left(\phi_{i}\left(q_{i}+\gamma\right) \boldsymbol{I}+\boldsymbol{U}(\gamma)\right),
\end{aligned}
$$

after multiplying (9) by $\left(q_{i}+\gamma\right) \boldsymbol{I}-\psi_{i}(-\boldsymbol{U}(\gamma))$ from the right and recalling definition (8). This is equivalent to

$$
\begin{aligned}
\gamma \boldsymbol{e}_{i}^{\prime}\left(\phi_{i}\left(q_{i}+\gamma\right) \boldsymbol{I}+\boldsymbol{U}(\gamma)\right)= & \boldsymbol{e}_{i}^{\prime} \psi_{i}(-\boldsymbol{U}(\gamma))\left(\phi_{i}\left(q_{i}+\gamma\right) \boldsymbol{I}+\boldsymbol{U}(\gamma)\right) \\
& -q_{i} \boldsymbol{e}_{i}^{\prime}\left(\phi_{i}\left(q_{i}+\gamma\right) \boldsymbol{I}+\boldsymbol{U}(\gamma)\right) \\
& +\sum_{k \neq i} q_{i k} \boldsymbol{e}_{k}^{\prime}\left(\begin{array}{c}
\boldsymbol{I}_{\sigma} \\
\boldsymbol{A}(\gamma)
\end{array}\right) \hat{F}_{i k}(\boldsymbol{U}(\gamma))\left(\phi_{i}\left(q_{i}+\gamma\right) \boldsymbol{I}+\boldsymbol{U}(\gamma)\right),
\end{aligned}
$$

since $\boldsymbol{U}(\gamma)$ and $\psi_{i}(-\boldsymbol{U}(\gamma))$ are commutative and $p_{i i}=0$. Using $-q_{i}=q_{i i}$ and multiplying by the inverse of $\phi_{i}\left(q_{i}+\gamma\right) \boldsymbol{I}+\boldsymbol{U}(\gamma)$ from the right, we obtain

$$
\gamma \boldsymbol{e}_{i}^{\prime}=\boldsymbol{e}_{i}^{\prime} \psi_{i}(-\boldsymbol{U}(\gamma))+\boldsymbol{e}_{i}^{\prime} \int_{0}^{\infty} \boldsymbol{Q} \circ \tilde{\boldsymbol{F}}(\mathrm{d} z)\left(\begin{array}{c}
\boldsymbol{I}_{\sigma} \\
\boldsymbol{A}(\gamma)
\end{array}\right) \mathrm{e}^{\boldsymbol{U}(\gamma) z},
$$

which is (5) for $i \in E_{\sigma}$. For the last term, recall definition (7) and note that the $(i, j)$ th entry of $\boldsymbol{Q} \circ \tilde{\boldsymbol{F}}$ is the measure $q_{i j} F_{i j}$.

For $i \in E_{-}$, we obtain from (10), after multiplying by $\left(q_{i}+\gamma\right) \boldsymbol{I}-\psi_{i}(-\boldsymbol{U}(\gamma))$ from the right,

$$
\boldsymbol{e}_{i}^{\prime} \boldsymbol{A}(\gamma)\left(\left(q_{i}+\gamma\right) \boldsymbol{I}-\psi_{i}(-\boldsymbol{U}(\gamma))\right)=\sum_{k \neq i} q_{i k} \boldsymbol{e}_{k}^{\prime}\left(\begin{array}{c}
\boldsymbol{I}_{\sigma} \\
\boldsymbol{A}(\gamma)
\end{array}\right) \hat{F}_{i k}(\boldsymbol{U}(\gamma)),
$$

which is equivalent to

$$
\gamma \boldsymbol{e}_{i}^{\prime} \boldsymbol{A}(\gamma)=\boldsymbol{e}_{i}^{\prime} \boldsymbol{A}(\gamma) \psi_{i}(-\boldsymbol{U}(\gamma))+q_{i i} \boldsymbol{e}_{i}^{\prime} \boldsymbol{A}(\gamma)+\sum_{k \neq i} q_{i k} \boldsymbol{e}_{k}^{\prime}\left(\begin{array}{c}
\boldsymbol{I}_{\sigma} \\
\boldsymbol{A}(\gamma)
\end{array}\right) \hat{F}_{i k}(\boldsymbol{U}(\gamma)),
$$

again using $q_{i i}=-q_{i}$. Now (7) and the definition of the Hadamard product $\boldsymbol{Q} \circ \tilde{\boldsymbol{F}}$ yield

$$
\gamma \boldsymbol{e}_{i}^{\prime} \boldsymbol{A}(\gamma)=\boldsymbol{e}_{i}^{\prime} \boldsymbol{A}(\gamma) \psi_{i}(-\boldsymbol{U}(\gamma))+\boldsymbol{e}_{i}^{\prime} \int_{0}^{\infty} \boldsymbol{Q} \circ \tilde{\boldsymbol{F}}(\mathrm{d} z)\left(\begin{array}{c}
\boldsymbol{I}_{\sigma} \\
\boldsymbol{A}(\gamma)
\end{array}\right) \mathrm{e}^{\boldsymbol{U}(\gamma) z},
$$

which is (5) for $i \in E_{-}$.

Example 2. Taking up the case treated in [1, Theorem 4.1], where $\gamma=0$ and there are no jumps (see Example 1), and writing $\boldsymbol{U}=\boldsymbol{U}(0)$ and $r_{i}=\mu_{i}$, (8) reduces to

$$
L_{i}(-\boldsymbol{U})=\frac{q_{i}}{\phi_{i}\left(q_{i}\right)} \cdot\left(\phi_{i}\left(q_{i}\right) \boldsymbol{I}+\boldsymbol{U}\right) \cdot\left(q_{i} \boldsymbol{I}-\psi_{i}(-\boldsymbol{U})\right)^{-1},
$$

while $\hat{F}_{i k}(\boldsymbol{U})=\boldsymbol{I}$, since there are no jumps. The cumulant function is

$$
\psi_{i}(-\boldsymbol{U})=\frac{\sigma_{i}^{2}}{2} \boldsymbol{U}^{2}-r_{i} \boldsymbol{U}
$$


In order to ease the comparison further, we shall use the same notation as in [1, Theorem 4.1], i.e. we set $\lambda_{i}=q_{i}$, and define

$$
\omega_{i}:=-\frac{r_{i}}{\sigma_{i}^{2}}+\sqrt{\frac{r_{i}^{2}}{\sigma_{i}^{4}}+\frac{2 \lambda_{i}}{\sigma_{i}^{2}}}, \quad \eta_{i}:=\frac{r_{i}}{\sigma_{i}^{2}}+\sqrt{\frac{r_{i}^{2}}{\sigma_{i}^{4}}+\frac{2 \lambda_{i}}{\sigma_{i}^{2}}} .
$$

Note that $\omega_{i}=\phi_{i}\left(q_{i}\right)$. The relations $\omega_{i} \eta_{i}=2 \lambda_{i} / \sigma_{i}^{2}$ and $\eta_{i}-\omega_{i}=2 r_{i} / \sigma_{i}^{2}$ lead to the factorisation

$$
\begin{aligned}
\lambda_{i} \boldsymbol{I}-\psi_{i}(-\boldsymbol{U}) & =-\frac{\sigma_{i}^{2}}{2} \boldsymbol{U}^{2}+r_{i} \boldsymbol{U}+\lambda_{i} \boldsymbol{I} \\
& =\frac{\sigma_{i}^{2}}{2}\left(-\boldsymbol{U}^{2}+\left(\eta_{i}-\omega_{i}\right) \boldsymbol{U}+\omega_{i} \eta_{i} \boldsymbol{I}\right) \\
& =\frac{\sigma_{i}^{2}}{2}\left(-\boldsymbol{U}+\eta_{i} \boldsymbol{I}\right)\left(\boldsymbol{U}+\omega_{i} \boldsymbol{I}\right),
\end{aligned}
$$

such that, if $\boldsymbol{U}+\omega_{i} \boldsymbol{I}$ is invertible, (13) becomes

$$
L_{i}(-\boldsymbol{U})=\frac{\lambda_{i}}{\omega_{i}} \frac{2}{\sigma_{i}^{2}}\left(\eta_{i} \boldsymbol{I}-\boldsymbol{U}\right)^{-1}=\eta_{i}\left(\eta_{i} \boldsymbol{I}-\boldsymbol{U}\right)^{-1} .
$$

Now (9) reads

$$
\boldsymbol{e}_{i}^{\prime} \boldsymbol{U}=\omega_{i} \sum_{k \in E} p_{i k} \boldsymbol{e}_{k}^{\prime} \eta_{i}\left(\eta_{i} \boldsymbol{I}-\boldsymbol{U}\right)^{-1}-\omega_{i} \boldsymbol{e}_{i}^{\prime}=\frac{2 \lambda_{i}}{\sigma_{i}^{2}} \sum_{k \in E} p_{i k} \boldsymbol{e}_{k}^{\prime}\left(\eta_{i} \boldsymbol{I}-\boldsymbol{U}\right)^{-1}-\omega_{i} \boldsymbol{e}_{i}^{\prime} .
$$

Since $\lambda_{i} p_{i k}=q_{i k}$ for $k \neq i, p_{i i}=0$, and $\lambda_{i}=-q_{i i}$, we can write

$$
\boldsymbol{e}_{i}^{\prime} \boldsymbol{U}=\boldsymbol{e}_{i}^{\prime}\left(\left(\boldsymbol{\Delta}_{2 / \sigma^{2}} \boldsymbol{Q}+\boldsymbol{\Delta}_{2 \lambda / \sigma^{2}}\right)\left(\eta_{i} \boldsymbol{I}-\boldsymbol{U}\right)^{-1}-\boldsymbol{\Delta}_{\omega}\right),
$$

where $\boldsymbol{\Delta}_{2 / \sigma^{2}}:=\operatorname{diag}\left(2 / \sigma_{1}^{2}, \ldots, 2 / \sigma_{m}^{2}\right)$, and $\boldsymbol{\Delta}_{2 \lambda / \sigma^{2}}$ and $\boldsymbol{\Delta}_{\omega}$ are defined analogously. Noting that $\boldsymbol{Q}$ is denoted by $\Lambda$ in [1], we see that this is the same form as in [1, Theorem 4.1].

Remark 4. By assumption, the phase process $\mathcal{g}$ is irreducible. This implies that no entry of any row $\boldsymbol{e}_{i}^{\prime} \boldsymbol{U}(\gamma)$ equals 0 , except in one case: if $E=E_{\sigma}$ and there are neither jumps nor a diffusion component (i.e. $\sigma_{i}=0, v_{i}=\mathbf{0}$, and $F_{i j}=\delta_{0}$ for all $j \in E$ ), then $F_{Y^{i}}=\delta_{0}$ and $L_{i}(-\boldsymbol{U}(\gamma))=\boldsymbol{I}_{\sigma}$. Hence,

$$
\sum_{k \in E} p_{i k} \boldsymbol{e}_{k}^{\prime} \int_{0}^{\infty} \mathrm{e}^{\boldsymbol{U}(\gamma) z} \mathrm{~d} \tilde{F}_{i k}(z) \int_{0}^{\infty} \mathrm{e}^{\boldsymbol{U}(\gamma) y} \mathrm{~d} F_{Y^{i}}(y) \boldsymbol{e}_{j}=\sum_{k \in E} p_{i k} \boldsymbol{e}_{k}^{\prime} \boldsymbol{I} \boldsymbol{e}_{j}=\sum_{k \in E} p_{i k} \delta_{k j}=p_{i j} .
$$

Thus, for such rows, we obtain $U_{i j}(\gamma)=\phi_{i}\left(q_{i}\right) p_{i j}$ for $i \neq j$ and $U_{i i}(\gamma)=-\phi_{i}\left(q_{i}+\gamma\right)$. Furthermore, the form of $\phi_{i}$ is particularly simple for such phases, namely, $\phi_{i}(s)=s / \mu_{i}$ for all $s \geq 0$. This means that the values for the $i$ th row of $\boldsymbol{U}(\gamma)$ are given immediately by

$$
U_{i i}(\gamma)=\frac{q_{i}+\gamma}{\mu_{i}} \quad \text { and } \quad U_{i j}(\gamma)=\frac{q_{i j}}{\mu_{i}} \quad \text { for all } j \in E
$$

Example 3. In the case of pure drifts in every phase (i.e. $\sigma_{i}=0$ and $v_{i}=\mathbf{0}$ for all $i \in E$ ) and no jumps upon phase changes (i.e. $F_{i k}=\delta_{0}$ for all $i, k \in E$ ), we obtain the fluid flow model 
without Brownian motion. In particular, for $\gamma=0$, Theorem 3.1 of [1] can be retrieved as follows. The fact that there are no jumps upon phase changes implies that $\hat{F}_{i k}(\boldsymbol{U}(\gamma))=\boldsymbol{I}_{\sigma}$ for all $i, k \in E$. From Remark 4 we know that $\psi_{i}(x)=\mu_{i} x$ and $\phi_{i}(y)=y / \mu_{i}$ for all $x, y \geq 0$. Thus,

$$
L_{i}(-\boldsymbol{U}(0))=\mu_{i} \cdot\left(\frac{q_{i}}{\mu_{i}} \boldsymbol{I}_{\sigma}+\boldsymbol{U}(0)\right) \cdot\left(q_{i} \boldsymbol{I}_{\sigma}+\mu_{i} \boldsymbol{U}(0)\right)^{-1}=\boldsymbol{I}_{\sigma} .
$$

Using this in (9) and noting that $p_{i i}=0$ and $q_{i}=-q_{i i}$, we obtain

$$
\boldsymbol{e}_{i}^{\prime} \boldsymbol{U}(0)=-\frac{q_{i}}{\mu_{i}} \boldsymbol{e}_{i}^{\prime}+\frac{q_{i}}{\mu_{i}} \sum_{k \in E} p_{i k} \boldsymbol{e}_{k}^{\prime}\left(\begin{array}{c}
\boldsymbol{I}_{\sigma} \\
\boldsymbol{A}(0)
\end{array}\right) \quad \text { for all } i \in E_{\sigma} .
$$

Since $-q_{i}=q_{i i}$ and $p_{i i}=0$, this can be written in a matrix form as

$$
\boldsymbol{U}(0)=\boldsymbol{\Delta}_{1 / \mu} \boldsymbol{Q}_{(\sigma, \sigma)}+\boldsymbol{\Delta}_{1 / \mu} \boldsymbol{Q}_{(\sigma,-)} \boldsymbol{A}(0),
$$

where $\boldsymbol{\Delta}_{1 / \mu}$ denotes the diagonal matrix with entries $1 / \mu_{i}, i \in E_{\sigma}$, and $\boldsymbol{Q}_{(\sigma, \sigma)}$ and $\boldsymbol{Q}_{(\sigma,-)}$ are the obvious blocks of the matrix $\boldsymbol{Q}$. This is the same as Equation (3.1) of [1], where the notation is $r_{i}=\mu_{i}, \Lambda=\boldsymbol{Q}, \alpha^{(-+)}=\boldsymbol{A}(0)$, and $U=\boldsymbol{U}(0)$. Equation (10) reduces to

$$
\boldsymbol{e}_{i}^{\prime} \boldsymbol{A}(0)=\sum_{k \neq i} q_{i k} \boldsymbol{e}_{k}^{\prime}\left(\begin{array}{c}
\boldsymbol{I}_{\sigma} \\
\boldsymbol{A}(0)
\end{array}\right)\left(q_{i} \boldsymbol{I}_{\sigma}+\mu_{i} \boldsymbol{U}(0)\right)^{-1} \quad \text { for all } i \in E_{-} .
$$

Since all $\mu_{i}$ are negative, we can write this in closed form as

$$
\boldsymbol{A}(0)=\boldsymbol{\Delta}_{1 /|\mu|}\left(\boldsymbol{Q}_{(-, \sigma)}+\boldsymbol{Q}_{(-,-)} \boldsymbol{A}(0)+\boldsymbol{\Delta}_{q} \boldsymbol{A}(0)\right)\left(\boldsymbol{\Delta}_{q /|\mu|}-\boldsymbol{U}(0)\right)^{-1},
$$

where again for a vector $\boldsymbol{v}$ the matrix $\boldsymbol{\Delta}_{\boldsymbol{v}}$ shall be diagonal with the same entries as $\boldsymbol{v}$. Using (14), we see that this is the same fixed point equation for $\boldsymbol{A}(0)$ as in [1, Theorem 3.1] for the matrix $\boldsymbol{\alpha}^{(-+)}$.

Theorem 2. The matrices $\boldsymbol{A}(\gamma)$ and $\boldsymbol{U}(\gamma)$ can be computed as the limit of the sequence $\left(\boldsymbol{A}_{n}, \boldsymbol{U}_{n}: n \geq 0\right)$, which is defined by the initial values $\boldsymbol{U}_{0}:=-\operatorname{diag}\left(\phi_{i}\left(q_{i}+\gamma\right)\right)_{i \in E_{\sigma}}$ and $\boldsymbol{A}_{0}:=\mathbf{0}$, and the iteration

$$
\boldsymbol{e}_{i}^{\prime} \boldsymbol{U}_{n+1}:=-\phi_{i}\left(q_{i}+\gamma\right) \boldsymbol{e}_{i}^{\prime}+\phi_{i}\left(q_{i}\right) \sum_{k \in E} p_{i k} \boldsymbol{e}_{k}^{\prime}\left(\begin{array}{c}
\boldsymbol{I}_{\sigma} \\
\boldsymbol{A}_{n}
\end{array}\right) \hat{F}_{i k}\left(\boldsymbol{U}_{n}\right) L_{i}\left(-\boldsymbol{U}_{n}\right)
$$

for all $i \in E_{\sigma}$ and

$$
\boldsymbol{e}_{i}^{\prime} \boldsymbol{A}_{n+1}:=\sum_{k \neq i} q_{i k} \boldsymbol{e}_{k}^{\prime}\left(\begin{array}{c}
\boldsymbol{I}_{\sigma} \\
\boldsymbol{A}_{n}
\end{array}\right) \hat{F}_{i k}\left(\boldsymbol{U}_{n}\right)\left(\left(q_{i}+\gamma\right) \boldsymbol{I}_{\sigma}-\psi_{i}\left(-\boldsymbol{U}_{n}\right)\right)^{-1}
$$

for $i \in E_{-}$and $n \in \mathbb{N}_{0}$.

Proof. Consider the number of phase changes in the sample paths until $\tau(x)<\infty$. The starting values $\boldsymbol{A}_{0}$ and $\boldsymbol{U}_{0}$ are correct if we consider only sample paths with no phase changes. The iteration formulae each add at least one more possible phase change to the set of considered sample paths. It follows, by induction on $n$, that the matrices $\boldsymbol{A}_{n}$ and $\boldsymbol{U}_{n}$ comprise at least all sample paths with up to $n$ phase changes. Hence, $\lim _{n \rightarrow \infty}\left(\boldsymbol{U}_{n}, \boldsymbol{A}_{n}\right)$ yields the correct result for $\boldsymbol{U}(\gamma)$ and $\boldsymbol{A}(\gamma)$, since there are only finitely many phase changes in almost all sample paths during the finite time interval $[0, \tau(x)]$. 
Remark 5. For a phase $i \in E_{\sigma}$ with $\sigma_{i}=0, \mu_{i}=1, v_{i}=\mathbf{0}$, and $F_{i j}=\delta_{0}$ for all $j \in E$, the singular case $\left(q_{i}+\gamma\right) \boldsymbol{I}-\psi_{i}\left(-\boldsymbol{U}_{0}\right)=\mathbf{0}$ arises. Remark 4 shows how to deal with this. We can compute the values for $\boldsymbol{e}_{i}^{\prime} \boldsymbol{U}(\gamma)$ beforehand and put them as initial values into $\boldsymbol{U}_{0}$, knowing that no iteration will change them.

\section{Positive jumps of phase type}

Now assume that the Lévy measures $v_{i}$ have a positive part (i.e. with support on $(0, \infty)$ ), which is a compound Poisson process of jump intensity $\lambda_{i}^{+}$and jump sizes of phase-type distribution with parameters $\left(\boldsymbol{\alpha}^{(i i)}, \boldsymbol{T}^{(i i)}\right)$ for $i \in E$. Without loss of generality, we assume that the distributions $\mathrm{PH}\left(\boldsymbol{\alpha}^{(i i)}, \boldsymbol{T}^{(i i)}\right)$ have no atom (which would be on zero anyway). Note that in this setting the processes $\mathcal{X}^{(i)}$ for $i \in E_{-}$are not necessarily negative subordinators anymore, but in general superpositions of a negative subordinator and a compound Poisson process with (positive) phase-type jumps.

Furthermore, we assume that a phase change from $i$ to $j$ may trigger a positive jump of distribution $\mathrm{PH}\left(\boldsymbol{\alpha}^{(i j)}, \boldsymbol{T}^{(i j)}\right)$ with probability $\lambda_{i j}^{+}$. Note that in connection with the spectrally negative part of the model described before this section the restriction $\lambda_{i j}^{+}+\lambda_{i j}^{-} \leq 1$ applies. Again, we may assume, without loss of generality, that the distributions $\mathrm{PH}\left(\boldsymbol{\alpha}^{(i j)}, \boldsymbol{T}^{(i j)}\right)$ have no atom, which means that a phase change from $i$ to $j$ without an accompanying jump occurs with probability $1-\lambda_{i j}^{+}-\lambda_{i j}^{-} \leq 1$. Denote the respective exit vectors by $\boldsymbol{\eta}^{(i j)}:=-\boldsymbol{T}^{(i j)} \mathbf{1}$, where 1 denotes the column vector (of appropriate dimension) with all entries being 1 .

Our aim is again an expression for the (matrix-valued) Laplace transform $\mathrm{E}\left(\mathrm{e}^{-\gamma \tau(x)}\right)$ of the first passage time $\tau(x):=\min \left\{t \geq 0: X_{t} \geq x\right\}$ for $x>0$. The main idea is to spread out the phase-type positive jumps as a succession of linear pieces (each with positive slope 1) of exponential duration; cf. [2] or [7]. Then the new process is again a MAP without positive jumps, to which we can apply the methods derived before. To account for the jump nature of the new phase-type upward movements, we simply do not discount the time, i.e. we treat these phases with $\gamma=0$. This is the same method as applied in [7] for a fluid flow model. Thus, we again obtain a phase-type form

$$
\mathrm{E}\left(\mathrm{e}^{-\gamma \tau(x)} \mid X_{0}=0\right)=\left(\begin{array}{c}
\boldsymbol{I}_{\sigma} \\
\boldsymbol{A}(\gamma)
\end{array}\right) \mathrm{e}^{\boldsymbol{U}(\gamma) x} \quad \text { for } x \geq 0,
$$

and we need to determine the matrices $\boldsymbol{A}(\gamma)$ and $\boldsymbol{U}(\gamma)$.

Denote the order of the distribution $\mathrm{PH}\left(\boldsymbol{\alpha}^{(i j)}, \boldsymbol{T}^{(i j)}\right)$, which is the dimension of the vector $\boldsymbol{\alpha}^{(i j)}$, by $m_{i j}$. We add the set

$$
E_{+}:=\left\{(i, j, k): i, j \in E, 1 \leq k \leq m_{i j}\right\}
$$

of new phases to the old phase space $E=E_{\sigma} \cup E_{-}$, and denote the new phase space by $E^{\prime}:=E_{+} \cup E$, where it is understood that $E \cap E_{+}=\varnothing$. By Remark 4, the entries of $\boldsymbol{U}(\gamma)$ for a jump phase $p \in E_{+}$can be computed immediately, i.e. without the iteration in Theorem 2 .

Define $\tilde{q}_{i}:=q_{i}+\lambda_{i}^{+}$and $\tilde{p}_{i i}:=\lambda_{i}^{+} / \tilde{q}_{i}$, and set $\lambda_{i i}^{+}:=1$ for all $i \in E_{\sigma} \cup E_{-}$. Furthermore, define $\tilde{p}_{i j}:=q_{i j} / \tilde{q}_{i}$ for all $i \neq j \in E_{\sigma} \cup E_{-}$. Let

$$
\hat{F}_{i j}(\boldsymbol{U}(\gamma)):=\int_{0}^{\infty} \mathrm{e}^{\boldsymbol{U}(\gamma) y} \mathrm{~d} \tilde{F}_{i j}(y)
$$


with $\tilde{F}_{i j}(0)=1-\lambda_{i j}^{-}-\lambda_{i j}^{+}$and $\tilde{F}_{i j}(\mathrm{~d} y)=\lambda_{i j}^{-} F_{i j}(-\mathrm{d} y)$, and

$$
L_{i}(-\boldsymbol{U}(\gamma)):=\frac{\tilde{q}_{i}}{\phi_{i}\left(\tilde{q}_{i}\right)} \cdot\left(\phi_{i}\left(\tilde{q}_{i}+\gamma\right) \boldsymbol{I}+\boldsymbol{U}(\gamma)\right) \cdot\left(\left(\tilde{q}_{i}+\gamma\right) \boldsymbol{I}-\psi_{i}(-\boldsymbol{U}(\gamma))\right)^{-1}
$$

for all $i, j \in E_{\sigma} \cup E_{-}$. Now we can state the following extension of Theorem 1 .

Theorem 3. Let $(\mathcal{X}, \mathcal{g})$ be a MAP with phase-type positive jumps. Then the matrices $\boldsymbol{U}(\gamma)$ and $\boldsymbol{A}(\gamma)$ satisfy the equations

$$
\boldsymbol{e}_{p}^{\prime} \boldsymbol{U}(\gamma)=\sum_{l=1}^{m_{i j}} T_{k l}^{(i j)} \boldsymbol{e}_{(i, j, l)}^{\prime}+\eta_{k}^{(i j)} \boldsymbol{e}_{j}^{\prime}\left(\begin{array}{c}
\boldsymbol{I}_{\sigma} \\
\boldsymbol{A}(\gamma)
\end{array}\right)
$$

for $p=(i, j, k) \in E_{+}$,

$$
\begin{aligned}
\boldsymbol{e}_{i}^{\prime} \boldsymbol{U}(\gamma)= & -\phi_{i}\left(\tilde{q}_{i}+\gamma\right) \boldsymbol{e}_{i}^{\prime}+\phi_{i}\left(\tilde{q}_{i}\right) \sum_{j \in E_{\sigma} \cup E_{-}} \tilde{p}_{i j} \boldsymbol{e}_{j}^{\prime}\left(\begin{array}{c}
\boldsymbol{I}_{\sigma} \\
\boldsymbol{A}(\gamma)
\end{array}\right) \hat{F}_{i j}(\boldsymbol{U}(\gamma)) L_{i}(-\boldsymbol{U}(\gamma)) \\
& +\phi_{i}\left(\tilde{q}_{i}\right) \sum_{j \in E_{\sigma} \cup E_{-}} \tilde{p}_{i j} \lambda_{i j}^{+} \sum_{k=1}^{m_{i j}} \alpha_{k}^{(i j)} \boldsymbol{e}_{(i, j, k)}^{\prime} L_{i}(-\boldsymbol{U}(\gamma))
\end{aligned}
$$

for all $i \in E_{\sigma}$, and

$$
\begin{aligned}
\boldsymbol{e}_{i}^{\prime} \boldsymbol{A}(\gamma)= & \sum_{j \in E_{\sigma} \cup E_{-}, j \neq i} q_{i j} \boldsymbol{e}_{j}^{\prime}\left(\begin{array}{c}
\boldsymbol{I}_{\sigma} \\
\boldsymbol{A}(\gamma)
\end{array}\right) \hat{F}_{i j}(\boldsymbol{U}(\gamma))\left(\left(\tilde{q}_{i}+\gamma\right) \boldsymbol{I}_{\sigma}-\psi_{i}(-\boldsymbol{U}(\gamma))\right)^{-1} \\
& +\sum_{j \in E_{\sigma} \cup E_{-}, j \neq i} q_{i j} \lambda_{i j}^{+} \sum_{k=1}^{m_{i j}} \alpha_{k}^{(i j)} \boldsymbol{e}_{(i, j, k)}^{\prime}\left(\left(\tilde{q}_{i}+\gamma\right) \boldsymbol{I}_{\sigma}-\psi_{i}(-\boldsymbol{U}(\gamma))\right)^{-1} \\
& +\lambda_{i}^{+} \sum_{k=1}^{m_{i i}} \alpha_{k}^{(i i)} \boldsymbol{e}_{(i, i, k)}^{\prime}\left(\left(\tilde{q}_{i}+\gamma\right) \boldsymbol{I}_{\sigma}-\psi_{i}(-\boldsymbol{U}(\gamma))\right)^{-1}
\end{aligned}
$$

for $i \in E_{-}$.

Proof. First note that the positive (phase-type) part of any Lévy measure $v_{i}$ is replaced by linear movements in the phases $(i, i, 1), \ldots,\left(i, i, m_{i i}\right)$. The rate to enter these phases is $\lambda_{i}^{+}$. Hence, we replace $q_{i}$ in Theorem 1 by $\tilde{q}_{i}=q_{i}+\lambda_{i}^{+}$. As a consequence, we change the transition probabilities $p_{i j}$ to $\tilde{p}_{i j}=p_{i j} q_{i} / \tilde{q}_{i}$ for all $i \neq j \in E_{\sigma} \cup E_{-}$.

The rows given in (15) follow from Remark 4, noting that, during movements in phases $p \in E_{+}$, we set $\gamma=0$.

The first two terms in (16) have exactly the same explanation as in the proof of Theorem 1. The last term in (16) subsumes all possibilities of a positive jump occurring from phase $i \in E_{\sigma}$. For $i=j$ in the sum, this is part of the Lévy measure $v_{i}$. Then the phase $(i, i, k)$ is entered with transition probability

$$
\frac{\lambda_{i}^{+}}{q_{i}+\lambda_{i}^{+}} \alpha_{k}^{(i i)}=\tilde{p}_{i i} \lambda_{i i}^{+} \alpha_{k}^{(i i)} \text { for } k=1, \ldots, m_{i i},
$$


as $\lambda_{i i}^{+}=1$ by definition. For $i \neq j$ in the sum, a positive jump happens as part of the phase change from $i$ to another phase $j \neq i$ with probability $\lambda_{i j}^{+}$. Then the phase $(i, j, k)$ is entered with transition probability

$$
\frac{q_{i}}{q_{i}+\lambda_{i}^{+}} p_{i j} \lambda_{i j}^{+} \alpha_{k}^{(i j)}=\tilde{p}_{i j} \lambda_{i j}^{+} \alpha_{k}^{(i j)} \quad \text { for } k=1, \ldots, m_{i j} .
$$

The consecutive matrix $\boldsymbol{L}(-\boldsymbol{U}(\gamma))$ plays the same role as in the first terms in (16) and is explained in the proof of Theorem 1.

Adapting the proof of Theorem 1 to the new rates $\tilde{q}_{i}$ and transition probabilities $\tilde{p}_{i j}$ explains the first term in (17). The last term in (17) mirrors the event that a jump happens due to the positive part of the Lévy measure $v_{i}$ in a descending phase $i \in E_{-}$. The phase $(i, i, k)$ is entered with transition probability $\tilde{p}_{i i} \alpha_{k}^{(i i)}$. This multiplied with the new rate $\tilde{q}_{i}$ yields $\lambda_{i}^{+} \alpha_{k}^{(i i)}$. The second term in (17) subsumes positive jumps upon a phase change from $i$ to another phase $j \neq i$. As above, the phase $(i, j, k)$ is entered with transition probability $\tilde{p}_{i j} \lambda_{i j}^{+} \alpha_{k}^{(i j)}$, which upon multiplication with $\tilde{q}_{i}$ yields $q_{i j} \lambda_{i j}^{+} \alpha_{k}^{(i j)}$. The common remaining factor $\left(\left(\tilde{q}_{i}+\gamma\right) \boldsymbol{I}_{\sigma}-\psi_{i}(-\boldsymbol{U}(\gamma))\right)^{-1}$ has the same explanation as in the proof of Theorem 1.

Example 4. The case of a Lévy process with positive jumps of phase type has been examined in [19]. If this Lévy process without the positive jumps is not a negative subordinator then $E_{-}=\varnothing$ and $E_{\sigma}=\{1\}$. There can be no phase changes within $E_{-} \cup E_{\sigma}$, such that the terms involving $F_{i j}$ do not apply. The frequency of the positive jumps is denoted by $\lambda>0$ in [19], such that $\tilde{q}_{1}=\lambda$. The number $\phi_{1}\left(\tilde{q}_{1}+\gamma\right)$ is denoted by $p$ in [19]. The phasetype distribution of the positive jumps has parameters $(\boldsymbol{\pi}, \boldsymbol{T})$ with dimension $d$, such that $E_{+}=\{(1,1,1), \ldots,(1,1, d)\}$ and $\alpha_{k}^{(1,1)}=\pi_{k}$ for $k \leq m_{11}=d$. Applying (15), we see that

$$
\boldsymbol{U}(\gamma)=\left(\begin{array}{cc}
\boldsymbol{T} & \boldsymbol{\eta} \\
u_{1} \cdots u_{d} & u_{d+1}
\end{array}\right)
$$

where $\boldsymbol{\eta}=-\boldsymbol{T} \mathbf{1}$ and the phases in $E_{+}$are listed in the rows before phase 1 . Thus, it remains to determine the numbers $u_{1}, \ldots, u_{d+1}$. Since $\tilde{p}_{11}=\lambda_{1}^{+} / \tilde{q}_{1}=\lambda / \lambda=1$ and $\lambda_{11}^{+}=1$ by definition, (16) yields, for $j=1, \ldots, d$,

$$
\begin{aligned}
u_{j} & =\phi_{1}\left(\tilde{q}_{1}\right) \tilde{\boldsymbol{\pi}} L_{1}(-\boldsymbol{U}(\gamma)) \boldsymbol{e}_{j} \\
& =\tilde{\boldsymbol{\pi}} \tilde{q}_{1} \cdot\left(\phi_{1}\left(\tilde{q}_{1}+\gamma\right) \boldsymbol{I}+\boldsymbol{U}(\gamma)\right) \cdot\left(\left(\tilde{q}_{1}+\gamma\right) \boldsymbol{I}-\psi_{1}(-\boldsymbol{U}(\gamma))\right)^{-1} \boldsymbol{e}_{j} \\
& =\tilde{\boldsymbol{\pi}} \lambda \cdot(p \boldsymbol{I}+\boldsymbol{U}(\gamma)) \cdot\left((\lambda+\gamma) \boldsymbol{I}-\psi_{1}(-\boldsymbol{U}(\gamma))\right)^{-1} \boldsymbol{e}_{j} \\
& =p \tilde{\boldsymbol{\pi}} \frac{\lambda}{\lambda+\gamma}(\lambda+\gamma) \cdot\left((\lambda+\gamma) \boldsymbol{I}-\psi_{1}(-\boldsymbol{U}(\gamma))\right)^{-1} \cdot(p \boldsymbol{I}+\boldsymbol{U}(\gamma)) p^{-1} \boldsymbol{e}_{j},
\end{aligned}
$$

where $\tilde{\pi}=\left(\pi_{1}, \ldots, \pi_{d}, 0\right)$. In [19] the notation $\mu_{j}:=\lambda \pi_{j} /(\lambda+\gamma), \mu:=\left(\mu_{1}, \ldots, \mu_{d}, 0\right)$, and

$$
\hat{A}(U):=(\lambda+\gamma)\left((\lambda+\gamma) \boldsymbol{I}-\psi_{1}(-U)\right)^{-1} \cdot(p \boldsymbol{I}+U) p^{-1}
$$

were used for every $j=1, \ldots, d$ and a matrix $\operatorname{argument} U=\mathbf{S}+\mathbf{s} \mu+$, where

$$
\mathbf{S}:=\left(\begin{array}{cc}
\boldsymbol{T} & \eta \\
\mathbf{0} & -p
\end{array}\right), \quad \mathbf{s}:=\left(\begin{array}{c}
0 \\
\vdots \\
0 \\
p
\end{array}\right), \quad \text { and } \quad \mu+:=\mu \hat{A}(\mathbf{S}+\mathbf{s} \mu+)
$$


see (7) and (8) of [19]. Hence, we obtain, for $j=1, \ldots, d$,

$$
u_{j}=p \mu \hat{A}(\mathbf{S}+\mathbf{s} \mu+) \boldsymbol{e}_{j} \quad \text { and } \quad u_{d+1}=p \mu \hat{A}(\mathbf{S}+\mathbf{s} \mu+) \boldsymbol{e}_{d+1}-p,
$$

which coincides with [19, Theorem 1 , case (A)]. In particular, $\boldsymbol{U}(\gamma)=\mathbf{S}+\mathbf{s} \mu+$.

Case (B) of [19] dealt with a Lévy process which without the positive jumps would be a negative subordinator. Here $E_{\sigma}=\varnothing, E_{-}=\{1\}$, and $E_{+}=\{(1,1,1), \ldots,(1,1, d)\}$. This means that we need to determine a $d \times d$ matrix $\boldsymbol{U}(\gamma)$ and a $d$-dimensional row vector $\boldsymbol{A}(\gamma)$. Again there can be no phase changes within $E_{-} \cup E_{\sigma}$ such that the terms involving $F_{i j}$ do not apply. Equation (15) yields

$$
\boldsymbol{U}(\gamma)=\boldsymbol{T}+\boldsymbol{\eta} \boldsymbol{A}(\gamma),
$$

where $(\boldsymbol{\pi}, \boldsymbol{T})$ denote the parameters of the phase-type distribution governing the jump sizes and $\boldsymbol{\eta}=-\boldsymbol{T} 1$. Thus, it remains to determine the row vector $\boldsymbol{A}(\gamma)$. Equation (17) yields

$$
\begin{aligned}
\boldsymbol{A}(\gamma) & =\lambda \boldsymbol{\pi}\left((\lambda+\gamma) \boldsymbol{I}-\psi_{1}(-\boldsymbol{U}(\gamma))\right)^{-1} \\
& =\frac{\lambda}{\lambda+\gamma} \boldsymbol{\pi} \cdot(\lambda+\gamma)\left((\lambda+\gamma) \boldsymbol{I}-\psi_{1}(-\boldsymbol{U}(\gamma))\right)^{-1},
\end{aligned}
$$

where the last line shows that $\boldsymbol{A}(\gamma)$ equals $\pi+$ in [19]. This coincides with [19, Theorem 1, case (B)].

Example 5. We shall derive the Laplace transform of the ruin time for the classical compound Poisson risk model. In this there is an initial risk reserve $u \geq 0$, the claim sizes and interclaim times are independent and have exponential distributions with parameters $\beta>0$ and $\lambda>0$, respectively. The rate of premium income is denoted by $c>0$. This model has been analysed in [15]. The net profit condition is $c / \lambda>1 / \beta$, which is equivalent to $\lambda / c \beta<1$.

In terms of the methods available from the present paper we consider a $\operatorname{MAP}(\mathcal{X}, \mathcal{g})$, which is defined as follows. Let the phase space be given by $E_{-}=\{1\}, E_{+}=\{(1,1,1)\}$, and $E_{\sigma}=\varnothing$. The parameters are given by $\sigma_{1}=0, \mu_{1}=-c, v_{1}=\mathbf{0}$, and

$$
\boldsymbol{Q}=\left(\begin{array}{cc}
-\lambda & \lambda \\
\beta & -\beta
\end{array}\right) \text {. }
$$

Then the ruin time for the compound Poisson model coincides with the first passage time $\tau(u)$ given that we start with $X_{0}=0$.

According to the definitions in Section 5, we set $\boldsymbol{T}^{(1,1)}=-\beta$ and $\boldsymbol{\alpha}^{(1,1)}=1$. We then obtain from Theorem 3 that $\boldsymbol{A}(\gamma)$ and $\boldsymbol{U}(\gamma)$ are scalars and

$$
\boldsymbol{U}(\gamma)=-\beta+\beta \boldsymbol{A}(\gamma) \quad \text { and } \quad \boldsymbol{A}(\gamma)=\lambda \cdot(\lambda+\gamma-c \boldsymbol{U}(\gamma))^{-1} .
$$

This yields, for $\boldsymbol{A}(\gamma)$, the two solutions

$$
\boldsymbol{A}(\gamma)=\frac{\lambda+\gamma+c \beta}{2 c \beta} \pm \sqrt{\frac{1}{4}\left(\frac{\lambda+\gamma}{c \beta}+1\right)^{2}-\frac{\lambda}{c \beta}},
$$

from which we take the smaller one in accordance with Theorem 2. After some elementary algebra, we obtain

$$
\begin{aligned}
\boldsymbol{A}(\gamma) & =\frac{1}{2 c \beta}\left(\lambda+\gamma+c \beta-\sqrt{(\lambda+\gamma+c \beta)^{2}-4 \lambda c \beta}\right) \\
& =\frac{1}{2 c \beta}\left(\lambda+\gamma+c \beta-\sqrt{(c \beta-(\lambda+\gamma))^{2}+4 \gamma c \beta}\right)
\end{aligned}
$$


and

$$
\boldsymbol{U}(\gamma)=\frac{1}{2 c}\left(\lambda+\gamma-c \beta-\sqrt{(c \beta-(\lambda+\gamma))^{2}+4 \gamma c \beta}\right) .
$$

Thus, $\boldsymbol{U}(\gamma)$ coincides with $-R$ in [15, Equation (4.24)], noting that our $\gamma$ is denoted by $\delta$ there. This in turn yields $\boldsymbol{A}(\gamma)=(\beta-R) / \beta$ and $(4)$, namely $\mathrm{E}\left(\mathrm{e}^{-\gamma \tau(u)}\right)=\boldsymbol{A}(\gamma) \mathrm{e}^{\boldsymbol{U}(\gamma) u}$, translates into

$$
\mathrm{E}\left(\mathrm{e}^{-\gamma \tau(u)}\right)=\frac{\beta-R}{\beta} \mathrm{e}^{-R u},
$$

which is Equation (5.38) of [15].

\section{Overall supremum and the stationary distribution of the reflected process}

The first passage times are closely related to other important characteristics such as suprema and the stationary distribution of the reflected process. This shall be described shortly in the present section. As a preparation, the following observation is useful. By definition,

$$
\begin{aligned}
\mathrm{E}\left(\mathrm{e}^{-\gamma \tau(x)} \mid X_{0}=0\right) & =\int_{0}^{\infty} \mathrm{e}^{-\gamma t} \mathrm{P}\left(\tau(x) \in \mathrm{d} t \mid X_{0}=0\right) \\
& =\int_{0}^{\infty} \gamma \mathrm{e}^{-\gamma t} \mathrm{P}\left(\tau(x) \leq t \mid X_{0}=0\right) \mathrm{d} t \quad \text { for all } x \geq 0,
\end{aligned}
$$

where the last equality is due to integration by parts. For the supremum process, $S_{t}:=$ $\sup \left\{X_{s}: s \leq t\right\}$, the relation

$$
\mathrm{P}\left(\tau(x) \leq t \mid X_{0}=0\right)=\mathrm{P}\left(S_{t} \geq x \mid X_{0}=0\right)
$$

holds for $x>0$. This implies that $\mathrm{E}\left(\mathrm{e}^{-\gamma \tau(x)} \mid X_{0}=0\right)=\mathrm{P}\left(S_{\mathcal{E}(\gamma)} \geq x \mid X_{0}=0\right)$ for all $x>0$, where $\mathcal{E}(\gamma)$ is an independent random variable with exponential distribution of rate $\gamma$. Hence, we obtain

$$
\mathrm{P}\left(S_{\mathcal{E}(\gamma)} \geq x \mid X_{0}=0\right)=\left(\begin{array}{c}
\boldsymbol{I}_{\sigma} \\
\boldsymbol{A}(\gamma)
\end{array}\right) \mathrm{e}^{\boldsymbol{U}(\gamma) x} \quad \text { for all } x>0 .
$$

Thus, the supremum before an exponential killing time $\mathscr{E}(\gamma)$ has a phase-type distribution with rate matrix $\boldsymbol{U}(\gamma)$. This generalises Lemma 2 .

Let $\boldsymbol{\pi}$ denote the stationary distribution of the phase process 9 , i.e. $\boldsymbol{\pi} \boldsymbol{Q}=\mathbf{0}$, where $\mathbf{0}$ is the row vector with all entries being 0 . Furthermore, define the drift of the Lévy process in phase $i$ by $m_{i}:=\mathrm{E}\left(X_{1}^{i}\right)$ and write $\boldsymbol{m}=\left(m_{i}\right)_{i \in E}$ as a column vector. If the mean drift $\boldsymbol{\pi} \boldsymbol{m}$ is negative then the overall supremum $S_{\infty}:=\sup \left\{X_{t}: t \geq 0\right\}$ is finite almost surely. In the limit $\gamma \rightarrow 0$ we obtain the following theorem.

Theorem 4. If the mean drift $\boldsymbol{\pi} \boldsymbol{m}$ is negative then the overall supremum $S_{\infty}$ has a phase-type distribution with parameters

$$
\left(\beta\left(\begin{array}{c}
\boldsymbol{I}_{\sigma} \\
\boldsymbol{A}(\gamma)
\end{array}\right), \boldsymbol{U}(0)\right)
$$

where $\beta$ is the distribution of $J_{0}$.

Now define the reflected process $X_{t}^{r}:=X_{t}-\inf \left\{X_{s}: s \leq t\right\}$. If $\pi \boldsymbol{m}<0$ then the process $\left(X^{r}, \mathcal{g}\right)$ has a stationary distribution. Denote its steady-state variables by $\left(X^{r}, J\right)$. Denote a time-reversed version of $(\mathcal{X}, \mathcal{g})$ by $(\tilde{X}, \tilde{g})$, where the generator matrix for $\tilde{g}$ is given by 
$\tilde{\boldsymbol{Q}}:=\boldsymbol{\Delta}_{1 / \pi} \boldsymbol{Q} \boldsymbol{\Delta}_{\pi}$ and $\tilde{X}$ has the same parameters as $\mathcal{X}$ but depends on $\tilde{\mathcal{g}}$. Finally, write $\tilde{S}_{\infty}:=\sup \left\{\tilde{X}_{t}: t \geq 0\right\}$. Then Proposition 2.1 of [1] yields

$$
\mathrm{P}\left(X^{r}>x, J=i\right)=\pi_{i} \mathrm{P}\left(\tilde{S}_{\infty}>x \mid \tilde{J}_{0}=i, \tilde{\boldsymbol{X}}_{0}=0\right)=\pi_{i} \boldsymbol{e}_{i}^{\prime}\left(\begin{array}{c}
\boldsymbol{I}_{\sigma} \\
\tilde{\boldsymbol{A}}(0)
\end{array}\right) \mathrm{e}^{\tilde{\boldsymbol{U}}(0) x} \mathbf{1},
$$

where $\tilde{\boldsymbol{U}}(0)$ and $\tilde{\boldsymbol{A}}(0)$ pertain to $(\tilde{\mathcal{X}}, \tilde{\boldsymbol{g}})$ and $\mathbf{1}$ denotes the column vector with all entries being 1 .

\section{Diagonalisation and the Asmussen-Kella martingale in the absence of negative subordinators}

One possibility to determine the Laplace transform of the first passage times $\tau(x)$ is the use of appropriate (vector-valued) martingales, as proposed in [4]. Choose $Y_{t}:=-\gamma t / \alpha$ in Theorem 2.1 of [4]. Then Equation (2.9) therein states that

$$
\boldsymbol{M}(\alpha, t)=\int_{0}^{t} \exp \left(\alpha Z_{s}\right) 1_{J_{s}} \mathrm{~d} s \cdot(\boldsymbol{F}(\alpha)-\gamma \boldsymbol{I})+\exp \left(\alpha Z_{0}\right) 1_{J_{0}}-\exp \left(\alpha Z_{t}\right) 1_{J_{t}}
$$

is a row vector-valued zero-mean martingale, where $Z_{t}:=X_{t}+Y_{t}$ for a $\operatorname{MAP}\left(X_{t}, J_{t}: t \geq 0\right)$ and the notation is $\boldsymbol{F}(\alpha)=\boldsymbol{K}(\alpha)=\operatorname{diag}\left(\psi_{i}(\alpha)\right)+\boldsymbol{Q} \circ \hat{\boldsymbol{F}}(-\alpha)$ (cf. definition (2)). If we can find complex numbers $\alpha_{1}, \ldots, \alpha_{m}$ and corresponding linearly independent vectors $\boldsymbol{h}^{1}, \ldots, \boldsymbol{h}^{m}$ such that

$$
\left(\boldsymbol{F}\left(\alpha_{j}\right)-\gamma \boldsymbol{I}\right) \boldsymbol{h}^{j}=\mathbf{0} \text { for all } j=1, \ldots, m,
$$

then the Laplace transform of $\tau(x)$ can be determined by optional stopping and solving a resulting system of linear equations. This approach has been applied to option pricing in [5] and to insurance risk in [6].

In this section we shall explore the relation between that approach and a diagonalisation of $\boldsymbol{U}(\gamma)$ for the special case that none of the phases governs a negative subordinator, i.e. $E_{-}=\varnothing$ or $E=E_{\sigma}$. Denote the moment generating function of $\tilde{F}_{i j}$ by $\hat{F}_{i j}(s)=\int_{0}^{\infty} \mathrm{e}^{s x} \mathrm{~d} \tilde{F}_{i j}(x)$ and, furthermore, define the matrix $\hat{\boldsymbol{F}}(s)=\left(\hat{F}_{i j}(s)\right)_{i, j \in E}$ for all complex numbers $s \in \mathbb{C}$ for which the entries $\hat{F}_{i j}(s)$ are defined.

Theorem 5. If there are complex numbers $s_{1}, \ldots, s_{m}$ with $\operatorname{Re}\left(s_{j}\right)>0$ for all $j \in E$ and respective linearly independent vectors $\boldsymbol{h}^{1}, \ldots, \boldsymbol{h}^{m}$ such that

$$
\boldsymbol{e}_{i}^{\prime}\left(\psi_{i}\left(s_{j}\right) \cdot \boldsymbol{I}+\boldsymbol{Q} \circ \hat{\boldsymbol{F}}\left(-s_{j}\right)-\gamma \boldsymbol{I}\right) \boldsymbol{h}^{j}=0 \text { for all } i, j \in E,
$$

then the matrix $\boldsymbol{U}(\gamma)$ has a diagonalisation of the form $\boldsymbol{U}(\gamma)=-\boldsymbol{H} \boldsymbol{\Delta}_{s} \boldsymbol{H}^{-1}$, where $\boldsymbol{H}=$ $\left(\boldsymbol{h}^{1}, \ldots, \boldsymbol{h}^{m}\right)$ and $\boldsymbol{\Delta}_{s}=\operatorname{diag}\left(s_{1}, \ldots, s_{m}\right)$.

Proof. It suffices to show that $\boldsymbol{U}(\gamma)=-\boldsymbol{H} \boldsymbol{\Delta}_{s} \boldsymbol{H}^{-1}$ solves (5). Since the vectors $\boldsymbol{h}^{1}, \ldots, \boldsymbol{h}^{m}$ build a base for $\mathbb{R}^{m}$, this is equivalent to

$\boldsymbol{e}_{i}^{\prime} \psi_{i}\left(\boldsymbol{H} \boldsymbol{\Delta}_{s} \boldsymbol{H}^{-1}\right) \boldsymbol{h}^{j}+\sum_{k \in E} q_{i k} \boldsymbol{e}_{k}^{\prime} \int_{0}^{\infty} \exp \left(-\boldsymbol{H} \boldsymbol{\Delta}_{s} \boldsymbol{H}^{-1} y\right) \mathrm{d} \tilde{F}_{i k}(y) \boldsymbol{h}^{j}=\gamma h_{i j} \quad$ for all $i, j \in E$,

where $h_{i j}$ is the $(i, j)$ th entry of $\boldsymbol{H}$.

The form of the $\psi_{i}$ shows that $\psi_{i}\left(\boldsymbol{H} \boldsymbol{\Delta}_{s} \boldsymbol{H}^{-1}\right)=\boldsymbol{H} \psi_{i}\left(\boldsymbol{\Delta}_{s}\right) \boldsymbol{H}^{-1}$. Denoting the $i$ th row vector of $\boldsymbol{H}$ by $\boldsymbol{h}_{i}$, this yields, for the first term,

$$
\boldsymbol{e}_{i}^{\prime} \psi_{i}\left(\boldsymbol{H} \boldsymbol{\Delta}_{s} \boldsymbol{H}^{-1}\right) \boldsymbol{h}^{j}=\boldsymbol{h}_{i} \psi_{i}\left(\boldsymbol{\Delta}_{s}\right) \boldsymbol{e}_{j}=\boldsymbol{h}_{i} \psi_{i}\left(s_{j}\right) \boldsymbol{e}_{j}=h_{i j} \psi_{i}\left(s_{j}\right)
$$


For the second term, we obtain

$$
\begin{aligned}
\sum_{k \in E} q_{i k} \boldsymbol{e}_{k}^{\prime} \int_{0}^{\infty} \exp \left(-\boldsymbol{H} \boldsymbol{\Delta}_{s} \boldsymbol{H}^{-1} y\right) \mathrm{d} \tilde{F}_{i k}(y) \boldsymbol{h}^{j} & =\sum_{k \in E} q_{i k} \boldsymbol{h}_{k} \int_{0}^{\infty} \exp \left(-\boldsymbol{\Delta}_{s} y\right) \mathrm{d} \tilde{F}_{i k}(y) \boldsymbol{e}^{j} \\
& =\sum_{k \in E} q_{i k} h_{k j} \int_{0}^{\infty} \exp \left(-s_{j} y\right) \mathrm{d} \tilde{F}_{i k}(y) \\
& =\boldsymbol{e}_{i}^{\prime}\left(\boldsymbol{Q} \circ \hat{\boldsymbol{F}}\left(-s_{j}\right)\right) \boldsymbol{h}^{j} \\
& =\gamma h_{i j}-\boldsymbol{e}_{i}^{\prime} \psi_{i}\left(s_{j}\right) \boldsymbol{h}^{j},
\end{aligned}
$$

where the last equality is due to the assumption stated in (19). Since $\boldsymbol{e}_{i}^{\prime} \psi_{i}\left(s_{j}\right) \boldsymbol{h}^{j}=h_{i j} \psi_{i}\left(s_{j}\right)$, the proof is complete.

Theorem 6. If the matrix $\boldsymbol{U}(\gamma)$ has a diagonalisation of the form $\boldsymbol{U}(\gamma)=-\boldsymbol{H} \boldsymbol{\Delta}_{s} \boldsymbol{H}^{-1}$, with $\boldsymbol{H}=\left(\boldsymbol{h}^{1}, \ldots, \boldsymbol{h}^{m}\right)$ and $\boldsymbol{\Delta}_{s}=\operatorname{diag}\left(s_{1}, \ldots, s_{m}\right)$, then $\operatorname{Re}\left(s_{j}\right)>0$ and

$$
\boldsymbol{e}_{i}^{\prime}\left(\psi_{i}\left(s_{j}\right) \cdot \boldsymbol{I}+\boldsymbol{Q} \circ \hat{\boldsymbol{F}}\left(-s_{j}\right)-\gamma \boldsymbol{I}\right) \boldsymbol{h}^{j}=0 \text { for all } i, j \in E .
$$

Proof. This follows by the same arguments as in the proof of Theorem 5, together with the observation that all the eigenvalues of $\boldsymbol{U}(\gamma)$ must have negative real part.

Remark 6. Kyprianou and Palmowski [17] used the Asmussen-Kella martingale as a base for their analysis (see Theorem 4 therein). However, it seems difficult to arrive from there to an explicit algorithm for computing $\boldsymbol{U}(\gamma)$. This is reflected in their observation that 'establishing an expression for $\Lambda(q)$ for spectrally negative MAPs is an open problem', where $\Lambda(q)$ is the crucial expression for determining $\mathrm{E}\left(\mathrm{e}^{-\gamma \tau(x)}\right)$ in Theorem 1 of [17]. A solution to this problem is proposed by Theorem 2 in the present paper.

\section{Numerical examples}

Example 6. The topic of the first example is the equivalence of the martingale approach and the diagonalisation of $\boldsymbol{U}(\gamma)$. We will first compute $\boldsymbol{U}(\gamma)$ via the Asmussen-Kella martingale and then via the iteration in Theorem 2. Set $E=\{1,2\}, q_{1}=9, q_{2}=21, \sigma_{i}=2$ for $i=1,2$, $\mu_{1}=0, \mu_{2}=-8, v_{i}=\mathbf{0}$, and $F_{i j}=\delta_{0}$ for $i, j \in E$. Furthermore, set $\gamma=2$.

For the martingale approach, we need to find $s \in \mathbb{C}$ with positive real part such that

$$
\operatorname{det}\left(\begin{array}{cc}
2 s^{2}-11 & 9 \\
21 & 2 s^{2}-8 s-23
\end{array}\right)=0 .
$$

The two values $s_{1}=1.5530$ and $s_{2}=6.1205$ satisfy these conditions. Two respective eigenvectors in (19) are $\boldsymbol{h}^{1}=(0.82452,0.56584)^{\top}$ and $\boldsymbol{h}^{2}=(-0.13942,0.99023)^{\top}$. With $\boldsymbol{H}=\left(\boldsymbol{h}^{1}, \boldsymbol{h}^{2}\right)$, this yields

$$
\boldsymbol{U}(2)=-\boldsymbol{H} \boldsymbol{\Delta}_{s} \boldsymbol{H}^{-1}=\left(\begin{array}{cc}
-1.95545 & 0.58644 \\
2.85833 & -5.71805
\end{array}\right) .
$$

A direct computation of $\boldsymbol{U}(2)$ via the iteration in Theorem 2 yields

$$
\boldsymbol{U}(2)=\left(\begin{array}{cc}
-1.95545 & 0.58643 \\
2.85832 & -5.71803
\end{array}\right),
$$

which may be regarded as the same result. 
Example 7. Let $\sigma_{i}^{2}=0$ and $\mu_{i}=1$ for all $i \in E$. The Lévy measures shall have the simple form $v_{i}(-\mathrm{d} x)=\lambda_{i} \eta_{i} \exp \left(-\eta_{i} x\right) \mathrm{d} x$, with some given parameters $\lambda_{i}, \eta_{i}>0$. If the parameters are chosen such that the process has a negative mean drift then the overall supremum $M_{\infty}$ will be finite almost surely and $\mathrm{P}\left(M_{\infty}>x\right)=\exp (\boldsymbol{U}(0) x)$; cf. Theorem 4. Since the time aspect here is neglected $(\gamma=0)$, we can compute $\boldsymbol{U}=\boldsymbol{U}(0)$ by a fluid flow model as well. This yields a numerical test.

First we specify the necessary ingredients for Theorem 2:

$$
\begin{gathered}
\psi_{i}(-\boldsymbol{U})=-\boldsymbol{U}+\lambda_{i}\left(\int_{0}^{\infty} \mathrm{e}^{\boldsymbol{U} x} \eta_{i} \exp \left(-\eta_{i} x\right) \mathrm{d} x-\boldsymbol{I}\right)=-\boldsymbol{U}+\lambda_{i}\left(\eta_{i}\left(\eta_{i} \boldsymbol{I}-\boldsymbol{U}\right)^{-1}-\boldsymbol{I}\right), \\
\phi_{i}\left(q_{i}\right)=\frac{\lambda_{i}-\eta_{i}+q_{i}}{2}+\sqrt{\left(\frac{\lambda_{i}-\eta_{i}+q_{i}}{2}\right)^{2}+\eta_{i} q_{i},} \\
L_{i}(-\boldsymbol{U})=\frac{q_{i}}{\phi_{i}\left(q_{i}\right)} \cdot\left(\phi_{i}\left(q_{i}\right) \boldsymbol{I}+\boldsymbol{U}\right) \cdot\left(q_{i} \boldsymbol{I}-\psi_{i}(-\boldsymbol{U})\right)^{-1} .
\end{gathered}
$$

Let there be $m=2$ phases, and let $q_{1}=1$ and $q_{2}=2$. Furthermore, set $\eta_{1}=3, \lambda_{1}=4$, $\eta_{2}=4$, and $\lambda_{2}=6$. Then Theorem 2 yields

$$
\boldsymbol{U}=\left(\begin{array}{cc}
-2.5801 & 1.4238 \\
3.5470 & -5.1301
\end{array}\right)
$$

after 15 iterations. However, the initial value $\boldsymbol{U}_{0}$ had to be slightly perturbed to a new value, $\boldsymbol{U}_{0}=-\boldsymbol{\Delta}_{\phi(q)}+[0,0.1 ; 0.1,0]$, in order to avoid a singular matrix in the first iteration (cf. Remark 5).

This result can be compared with the following fluid flow model (see [1]). Set $E_{+}=\{1,2\}$, $E_{-}=\{3,4\}, r_{1}=r_{2}=1, r_{3}=r_{4}=-1$, and

$$
\boldsymbol{\Lambda}=\left(\begin{array}{cccc}
-5 & 1 & 4 & 0 \\
2 & -8 & 0 & 6 \\
3 & 0 & -3 & 0 \\
0 & 4 & 0 & -4
\end{array}\right)
$$

After 30 iterations, according to Theorem 3.1 of [1], the obtained values for $\boldsymbol{\alpha}^{(-+)}$and $\boldsymbol{U}=$ $\boldsymbol{T}^{(++)}+\boldsymbol{T}^{(+-)} \boldsymbol{\alpha}^{(-+)}$are

$$
\boldsymbol{\alpha}^{(-+)}=\left(\begin{array}{cc}
0.60496 & 0.10594 \\
0.25783 & 0.47832
\end{array}\right) \quad \text { and } \quad \boldsymbol{U}=\left(\begin{array}{cc}
-2.5802 & 1.4238 \\
3.5470 & -5.1301
\end{array}\right) \text {, }
$$

which can be regarded as the same result.

Example 8. Again, set $\gamma=0$ and $\boldsymbol{U}=\boldsymbol{U}(0)$. In the previous example the simple form of the Lévy measure led to a closed-form expression of the matrices $\psi_{i}(-\boldsymbol{U})$, which greatly simplified numerical evaluations. In the case of phase-type jumps (say with parameters $\boldsymbol{\alpha}^{i}, \boldsymbol{T}^{i}$, and exit vector $\left.\eta^{i}=-\boldsymbol{T}^{i} \mathbf{1}\right)$ such a closed form is not obvious, and we need to evaluate the integrals

$$
\int_{0}^{\infty} \mathrm{e}^{\boldsymbol{U} x} \boldsymbol{\alpha}^{i} \exp \left(\boldsymbol{T}^{i} x\right) \boldsymbol{\eta}^{i} \mathrm{~d} x
$$

numerically. Furthermore, the inverse functions $\phi_{i}$ need to be evaluated numerically. 
This example serves to demonstrate that this is possible from the view point of numerical stability. Again, we let $\sigma_{i}^{2}=0$ and $\mu_{i}=1$ for all $i \in E$. The Lévy measures shall have the form $v_{i}(-\mathrm{d} x)=\lambda_{i} \boldsymbol{\alpha}^{i} \exp \left(\boldsymbol{T}^{i} x\right) \eta^{i} \mathrm{~d} x$. Let there be $m=2$ phases, and let $q_{1}=1$ and $q_{2}=2$. Furthermore, set $\lambda_{1}=4$ and $\lambda_{2}=6$ as well as

$$
\boldsymbol{T}^{1}=\left(\begin{array}{cc}
-6 & 6 \\
0 & -6
\end{array}\right) \quad \text { and } \quad \boldsymbol{T}^{2}=\left(\begin{array}{cc}
-8 & 8 \\
0 & -8
\end{array}\right)
$$

with $\boldsymbol{\alpha}^{1}=\boldsymbol{\alpha}^{2}=(1,0)$. After 15 iterations, according to Theorem 2 , starting with $\boldsymbol{U}_{0}=\boldsymbol{\Delta}_{\phi(q)}$, we arrive at

$$
\boldsymbol{U}=\left(\begin{array}{cc}
-2.9148 & 1.4329 \\
3.6593 & -5.7093
\end{array}\right)
$$

Here the integration has been performed in a naive manner by a Riemann upper sum with step size $10^{-4}$ until the tail is bounded by $10^{-4}$. The inverse function can be determined easily, since the functions $\psi_{i}:\left[\phi_{i}(0), \infty\right) \rightarrow[0, \infty)$ are strictly increasing. This has been done to an accuracy of $10^{-5}$.

This result can again be compared against a fluid flow case. Its specification is $E_{+}=\{1,2\}$, $E_{-}=\{3,4,5,6\}, r_{1}=r_{2}=1, r_{i}=-1$ for $i \in E_{-}$, and

$$
\Lambda=\left(\begin{array}{cccccc}
-5 & 1 & 4 & 0 & 0 & 0 \\
2 & -8 & 0 & 0 & 6 & 0 \\
0 & 0 & -6 & 6 & 0 & 0 \\
6 & 0 & 0 & -6 & 0 & 0 \\
0 & 0 & 0 & 0 & -8 & 8 \\
0 & 8 & 0 & 0 & 0 & -8
\end{array}\right)
$$

After 30 iterations, according to Theorem 3.1 of [1], the values for the matrices $\boldsymbol{\alpha}^{(-+)}$and $\boldsymbol{U}=\boldsymbol{T}^{(++)}+\boldsymbol{T}^{(+-)} \boldsymbol{\alpha}^{(-+)}$are

$$
\boldsymbol{\alpha}^{(-+)}=\left(\begin{array}{cc}
0.521216 & 0.108179 \\
0.708560 & 0.086688 \\
0.276425 & 0.381737 \\
0.202654 & 0.604703
\end{array}\right) \quad \text { and } \quad \boldsymbol{U}=\left(\begin{array}{cc}
-2.9151 & 1.4327 \\
3.6586 & -5.7096
\end{array}\right)
$$

Given the crudeness of the numerical integration procedure, the accuracy is reasonable. However, the 30 iterations for the fluid flow case were computed much quicker than the 15 iterations for the jump case, which of course is due to simpler iteration rules.

\section{References}

[1] Asmussen, S. (1995). Stationary distributions for fluid flow models with or without Brownian motion. Stoch. Models 11, 1-20.

[2] Asmussen, S. (1995). Stationary distributions via first passage times. In Advances in Queueing: Theory, Methods, and Open Problems, ed. J. Dshalalow, CRC Press, Boca Raton, FL, pp. 79-102.

[3] Asmussen, S. (2003). Applied Probability and Queues. Springer, New York.

[4] Asmussen, S. and Kella, O. (2000). A multi-dimensional martingale for Markov additive processes and its applications. Adv. Appl. Prob. 32, 376-393.

[5] Asmussen, S., Avram, F. And Pistorius, M. (2004). Russian and American put options under exponential phase-type Lévy models. Stoch. Process. Appl. 109, 79-111.

[6] Badescu, A. AND Breuer, L. (2008). The use of vector-valued martingales in risk theory. Blätter DGVFM 29, 1-12.

[7] BAdescu, A. et al. (2005). Risk processes analyzed as fluid queues. Scand. Actuarial J. 2005, 127-141. 
[8] Bertoin, J. (1996). Lévy Processes. Cambridge University Press.

[9] Bingham, N. (1975). Fluctuation theory in continuous time. Adv. Appl. Prob. 7, 705-766.

[10] ÇInlar, E. (1972). Markov additive processes. I, II. Z. Wahrscheinlichkeitsth. 24, 85-121.

[11] Dieker, A. (2006). Applications of factorization embeddings for Lévy processes. Adv. Appl. Prob. 38, 768-791.

[12] Dieker, A. (2006). Extremes and fluid queues. Doctoral Thesis. Universiteit van Amsterdam.

[13] Ezhov, I. AND SKorokhod, A. (1969). Markov processes with homogeneous second component. I. II. Theory. Prob. Appl. 14, 1-13.

[14] Ezhov, I. And Skorokнod, A. (1969). Markov processes with homogeneous second component. II. Theory. Prob. Appl. 14, 652-667.

[15] Gerber, H. ANd Shiu, E. (1998). On the time value of ruin. N. Amer. Actuarial J. 2, 48-78.

[16] JaCobSEn, M. (2005). The time to ruin for a class of Markov additive risk processes with two-sided jumps. $A d v$. Appl. Prob. 37, 963-992.

[17] Kyprianou, A. AND Palmowski, Z. (2008). Fluctuations of spectrally negative Markov additive processes. (Lecture Notes Math. 1934), Séminaire de Probabilités XLI, Springer, Berlin.

[18] Miyazawa, M. and Takada, H. (2002). A matrix-exponential form for hitting probabilities and its application to a Markov-modulated fluid queue with downward jumps. J. Appl. Prob. 39, 604-618.

[19] Mordecki, E. (2002). The distribution of the maximum of a Lévy process with positive jumps of phase-type. Theory Stoch. Process. 8, 309-316.

[20] Pistorius, M. (2006). On maxima and ladder processes for a dense class of Lévy process. J. Appl. Prob. 43, 208-220. 